(Aus dem physiologischen Institut der Universität Strassburg i. Els.)

\title{
Ein neuer Beweis für die leitende Funktion der Neurofibrillen, nebst Bemerkungen über die Reflexzeit, Hemmungszeit und Latenzzeit des Muskels beim Blutegel.
}

Von

\section{Albrecht Bethe.}

(Mit 15 Textfiguren.)

Inhaltsübersicht.

Fragestellung ..................... 1

Literatur. . . . . . . . . . . . . . . . . . . 6

Untersuchungsobjekt und Kritik seiner Zulässigkeit . . . . . . . . . 9

Methodik . . . . . . . . . . . . . . 11

Versuche ..................... . . . . 15

Reflexlatenz und Muskellatenz.............. 15

Die Abhängigkeit der Latenzzeiten von der Höhe des Tonus . . . . . 19

Hemmungslatenz und Reflexlatenz ............. 23

Bestimmungen der Übertragungszeit bei verschiedenen Längen desselben

Banchmarkabschnittes .............26

Kritik der Versuche von Jenkins und Carison......... 30

Schlussbetrachtungen . . . . . . . . . . . . . . 32

Resultate . . . . . . . . . . . . . . . 35

\section{Fragestellung.}

Die Nerven aller Tiere sind in mehr oder weniger hohem Grade dehnbar und nehmen auch unter physiologischen Verbältnissen verschiedene Längen an. Diese physiologischen Längenveränderungen der Nerven sind am grössten bei den Tieren; welche eines festen inneren oder äusseren Skeletts entbehren (Mollusken und Würmer). Ein Blutegel ist z. B. bei physiologisch grösster Länge $3-3^{1 / 2}$ mal so lang als bei stärkster Kontraktion, und die durch den ganzen Körper ausgespannte Bauchganglienkette macht diese Längen- 
veränderungen fast vollkommen mit. Bei gestrecktem Körper ist die Bauchganglienkette gradlinig, lang und dünn; bei der Kontraktion bleibt sie gradlinig und wird kurz und dick, genau wie ein vorher gestreckter Gummifaden.

Man kann sich hiervon leicht durch Präparation der lebenden Tiere in verschiedenen Kontraktionszuständen oder an Schnitten von Tieren, die in verschieden starker Kontraktion fixiert wurden (A páthy), überzengen. Nur bei allerstärkster Kontraktion findet man bisweilen, aber durchaus nicht immer, eine schwache Schlängelung des Bauchmarks. Der in seinen Dimensionen variable Teil des Bauchmarks sind die zwischen den Ganglien ausgespannten Längskommissuren (Konnektive), welche in der Hauptsache aus parallel verlaufenden Nervenfasern bestehen. In jedem Nerv und jedem Konnektiv verlaufen lange Muskelfasern(A pá th y), welche wohl die Verkürzung unterstützen. Ob die Nervenstränge sich ohne diese Muskelfasern zu verkürzen imstande sind, ist nicht bekannt. Nach dem Herauspräparieren aus dem Körper zeigt sich die Verkürzungsfähigkeit der Nerven und Konnektive stets stark herabgesetzt. Wahrscheinlich wird also die Verkürzung der Nerven durch die Verkürzung des umliegenden Gewebes unterstïtzt werden.

Ebenso wie die ganzen Nervenstränge, so verändern aucb die in ihnen enthaltenen Nervenfasern bei den verschiedenen Kontraktionszuständen ihre Länge. Sie legen sich bei der Verkürzung des Nerven nicht innerhalb desselben in Wellenlinien, wie es ja sein könnte und bei manchen Tierarten tatsächlich nebenher vorkommt (Säuger), sondern sie werden selber kurz und dick und bleiben geradlinig. Am einfachsten überzeugt man sich hiervon an vitalgefärbten Methylenblaupräparaten. Im herauspräparierten Konnektiv mit einigen gefärbten Nervenfasern sind diese geradlinig, trotzdem das Konnektiv verkürzt ist. Zieht man das Konnektiv mit Nadeln in die Länge, so werden die gefärbten Fasern dünn und lang, werden aber wieder kürzer und dicker, wenn man mit dem Zug nachlässt. Noch deutlicher, weil die Längendifferenzen viel grösser sind, sieht man dies an gegefärbten Schnitten durch Tiere, welche in verschiedenen Kontraktionszuständen fixiert worden waren [A páth $\left.{ }^{1}\right)$ ].

Ganz anders verhalten sich die in den Nervenfasern enthaltenen Neurofibrillen. Diese verändern ihre Länge mit den Längenveränd erungen des N erven nicht; bei stärkster physiologischer Streckung der Nerven resp. Konnektive sind die Neurofibrillen nahezu geradlinig (Fig. 1A). Je stärker der Nerv

1) A páthy, Mitteil. d. zool. Station zu Neapel Bd. 12 S. 540 ff. 1897. 
sich verkürzt, desto welliger werden die Fibrillen innerhalb der geradlinig konturierten Nervenfasern gefunden [Apáthy $\left.\left.{ }^{1}\right)\right]$. (Siehe Fig. $1 B$ und $C$.)

Die Deutlichkeit der Fibrillen ist in den Figuren nicht übertrieben. Die Fibrillen treten in den Präparaten Apáthys (und auch in den nach meiner Methode hergestellten) trotz ihrer Feinheit mit einer solehen Schärfe und Deutlichkeit hervor, dass man unwillkürlich daran denkt, sie mit Drähten zu vergleichen. - Die

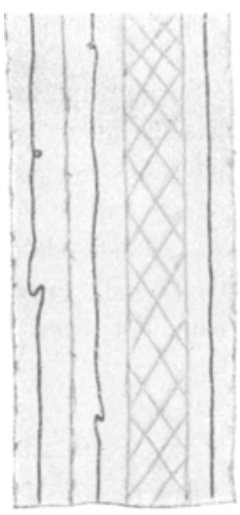

A.

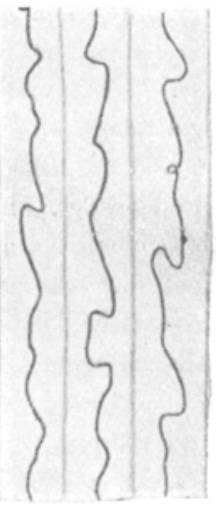

$B$.

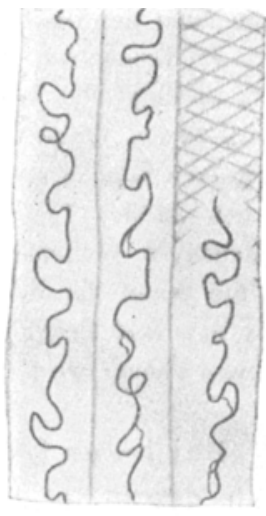

C.

Fig. 1. Drei Längsschnitte durch Konnektive vom Blutegel in verschiedenen Dehnungszuständen. (Skizzen nach Originalpräparaten von Prof. A páthy. Vergrösserung ca. 1200). A ganz gestreckt (physiologisehe Maximallânge). $B$ halb gestreckt (mittlerer Kontraktionszustand des Tieres). $C$ nicht gestreckt (starker Kontraktionszustand). Die abgebildeten Nervenfasern enthalten nur je eine dicke Neurofibrille, welche, wie in den Präparaten, ganz dunkel gehalten ist (blauschwarz). Die gradlinigen Konturen der Nervenfasern werden durch die in den Präparaten rötlich gefärbten "Gliascbeiden" dargestellt. Diese Scheiden bestehen aus spiralig um die Nervenfasern verlaufenden Gliafasern. In $A$ und $C$ sind solche Gliascheiden ${ }^{2}$ ) in der Aufsicht dargestellt, und man erkennt hier aus dom Winkel, in dem sich die Fasern überkrenzen, den verschiedenen Grad der Debmung der Nervenfasern. Die Perifibrillärsubstanz, das Nervenfaserplasma, ist in dea Präparaten ungefärbt.

1) Mitteil. d. zool. Station zu Neapel Bd. 12 S. 526 ff. 1897.

2) Bei den nach meiner Methode hergestellten Präparaten sind die Gliascheiden ungefärbt, so dass sie zwar die Verschiedenheit der Fibrillen im gedehuten und ungedehnten Zustand des Nerven sehr deutlich erkennen lassen, aber nichts über die jeweilige Form der ganzen Nervenfaser aussagen, worauf es hier besonders ankommt. Auch in den A páthly'schen Präparaten ist die Glia nicht immer mitgefärbt, so dass eine besondere Auswahl getroffen werden musste, um zu gleicher Zeit Kontur der Fasern und Gestalt der Fibrillen zu zeigen. Bei den Abbildungen ist mehr Wert auf das Charakteristische als auf die Einzelheiten gelegt. Die Schärfe der Bilder konnte bei der Art des Reproduktionsverfahrens nicht wiedergegeben werden. Ich verweise deswegen auf die $A b-$ bildungen Apáthys. 
immer noch hin und wieder vorgebrachte Meinung, die Neurofibrillen seien ein Kunstprodukt, kann ich besonders im Hinblick auf die Wirbellosen nicht recht ernst nehmen. Ein gewisser Skeptizismus gégenüber histologischen Befunden ist ja gewiss durchaus angebracht, wenn man bedenkt, wás für versichiedenartige Strukturen man durch: Gerinnung aus klarfiltrierten Eiweisslösungen gewinnen kann. Derartig lange, vollkommen glatte Fäden hat man aber bisher unter keinen Umiständen durch Gerinnuing erzielen können. Besonders die von A páthy entdeckten vollkommeñ gesetzmässigen Beziehungen zwischen Dehnungszustand der Nerven und der Schlängelung der Fibrillen lassen sich vom Gesichtspunkt des Gerinnungsproduktes schwerlich erklären.

$\mathrm{Zu}$ Demonstrationszwecken kann man sich leicht ein Modell einer Nervenfaser in folgender Weise herstellen: Man nimmt eine enggewickelte Spiralfeder von $2-4 \mathrm{~cm}$ Weite und etwa $50 \mathrm{~cm}$ Länge und bringt in dieselbe einen dünnen schwarzen Gummischlauch von ca. $100 \mathrm{~cm}$ Länge. Die Enden des Schlauches werden an den Enden der Spirale befestigt. In der Ruhelage legt sich der Schlauch in Wellenlinien oder korkzieherartig oder auch mit Schleifenbildung an die Wand der Spirale an. Zieht man die Spirale in die Länge, so erbält man das Bild der gestreckten Nervenfaser, indem die Kontur der Faser (die Spirale) dünner wird und die Fibrille im Innern sich streckt.

Bei jeder Längenveränderung des Nerven verändern. also die Nervenfasern ihre Länge in demselben Verhältnis. Die Länge der Fibrillen bleibt aber innerhalb der physiologischen Dehnungsgrenzen konstant.

Hieraus ergibt sich folgende Fragestellung: Ist das Nervenfaserplasma (die Perifibrillärsubstanz A páthy's oder das Hyaloplasma Leydig's) das Leitende im Nerven, so muss - eine gleichmässige Molekularstruktur des Plasmas vorausgesetzt - die "Übertragungszeit", d. h. die Zeit, in welcher der Reiz durch ein gegebenes Nervenstück fortgeleitet wird, bei Dehnung des Nervenstückes im gleichen Verhältnis zunehmen, weil der plasmatische Teil der Nervenfaser bei der Dehnung seine Länge verändert. Sind aber die Fibrillen das leitende Element im Nerven, so muss die Übertragungszeit für jede physiologische Länge des Nervenstückes gleich gross sein, weil dieselben bei der Dehuung des Nerven ihre Länge nicht verändern. - Wenn man von der üblichen Bestimmung der Leitungsgeschwindigkeit im Nerven ausgeht, kann man die Frage auch folgendermaassen formulieren: Ist das Nervenfaserplasma das Leitende; so muss die Leitungsgeschwindigkeit bei verschiedenen Längen des. gleichen Nervenstückes konstant sein. Wenn andererseits die Fibrillen das leitende Element im Nerven repräsentieren oder wenigstens bei 
der Leitung eine ausschlaggebendê Rolle spielen, so muss die Leitungsgeschwindigkeit in einem gegebenen Nervenstück proportional seiner jeweiligen . Länge sein.

Diese Fragestellung hat ihre Gültigkeit für jede der zurzeit herrschenden Nerventheorien. Ob man der alten Verbrennungstheorie huldigt und in der Nervenleitung eine fortschreitende Zersetzung sieht, oder sich den Nerven als Kernleiter (Hermann, Boruttau, Cremer) mit Kapazität und Selbstinduktion (Hermann) vorstellt, oder den Leitungsprozess als elektrochemischen Vorgang (Strong, Mathews, Loeb, Macdonald u. a.) oder als Kolloidvorgang (Hö ber) auffasst, stets wird, wenn nicht neue Annahmen gemacht werden, eine wirkliche Verlängerung des Leitungsweges eine Verlängerung der Übertragungszeit nach sich ziehen müssen. Fehlt im Experiment diese Verlängerung der Übertragungszeit bei Dehnung des reizleitenden Gewebes, so wird mau in diesem nach Elementen suchen, deren Länge konstant bleibt, und diese als das Leitende ansehen. Erst, wenn solche Elemente von konstanter Länge nicht auffindbar wären, und dieser nächstliegende Weg der Erklärung infolgedessen ungangbar würde, erst dann würde man sich nach anderen auf jeden Fall komplizierteren und gezwungeneren Frklärungsmöglichkeiten umsehen.

Zunächst ist also zu untersuchen, ob eine Veränderung der Übertragungszeit bei Dehnung des Nerven zu beobachten ist oder nicht. Das Prinzip der Versuche ist von vornherein gegeben: ein möglichst dehnbarer Nerv, dessen histologische Verbăltnisse genau bekannt sein müssen, wird nacheinander an zwei möglichst weit voneinander entfernten Stellen einmal im Stadium grösster Verkürzung, das andere Mal bei grösster physiologischer Dehnung gereizt. Die Differenz von je zwei zusammengehörigen Latenzen des zugehörigen Muskels gibt die Übertragungszeit ${ }^{1}$ ). Diese muss im Verhältnis der Längen des Nervenstückes stehen, wenn das Leitende in seiner Länge ebenso variiert wie der ganze Nerv; die Übertragungszeit muss aber konstant bleiben, wenn die Länge des Leitenden unverändert bleibt.

1) Natürlich könnte man die Übertragungszeiten ebensogut mit Hilfe der negativen Stromesschwankung oder des Aktionsstromes ermitteln; jedoch dürfte dies technisch auf einige Schwierigkeiten stossen, da die in Betracht kommenden Nerven in der Regel sehr dünn sind und durch das Herauspräparieren in ihrer Verkürzungsfähigkeit geschädigt werden. 
Von den uns bekannten und für die Leitung in Anspruch genommenen Gewebselementen trifft das erstere für das Nervenfaserplasma (die Perifibrillärsubstanz) zu, das letztere nur für die Neurofibrillen. Da wir in der Wissenschaft nur von dem zu handeln haben, über das wir etwas wissen, so besteht hier also nur die Alternative: Perifibrillärsubstanz oder Fibrille.

\section{Literatur.}

Auf die bisher für die leitende Funktion der Neurofibrillen aufgeführten Beweise brauche ich hier nicht einzugehen, da ich dieselben in meinem Buch ${ }^{1}$ ) eingehend besprochen habe.

Bestimmungen der Übertragungszeit bei gedehnten Nerven oder Kommissuren liegen bisher nur von Jenkins und Carls on ${ }^{2}$ ) und Carlson allein ${ }^{3}$ ) vor. Dass diesen Autoren die oben präzisierte Fragestellung bei ihren Untersuchungen vorgesehwebt hat, ist aus der gemeinsamen Publikation nicht ersichtlich. Dagegen findet sich in der Arbeit Carlson's eine dahin gehende Andeutung. Die Untersuchungen scheinen aus den günstigen Arbeitsobjekten und nicht aus einem bestimmten Problem herausgewachsen zu sein.

Als Objekt der ersten Arbeit diente der Pedalnerv einer Nacktschnecke (Ariolinax Columbianus), welcher auch im lebenden Tier grossen Längenveränderungen unterworfen ist. Um einen direkt motorischen Nerven handelt es sich hier höchstwahrseheinlich nicht, sondern um eine Kommissur, welche zwischen dem Pedalganglion und einem an der Peripherie gelegenen Nervennetz mit eingestreuten Ganglienzellen ausgespannt ist ${ }^{4}$ ). Der Nerv wurde nacheinander dicht am Muskel und weit vom Muskel entfernt gereizt. Die Differenz der Latenzzeiten ergab die Übertragungszeit. Diese wurde bestimmt bei grösster Verkürzung und bei grösster durch Zug bewirkter Verlängerung des freipräparierten Nerven.

Aus der Übertragungszeit und der jeweiligen Länge des Nervenstücks wird die Leitungsgeschwindigkeit für jeden Fall berechnet. Es ergibt sich aus der statistischen Zusammenstellung, dass die Leitungsgeschwindigkeit bei einfacher und ungefähr doppelter Länge

1) Bethe, Allgem. Anat. u. Physiol. des Nervensystems S. 19, 51, $261 \mathrm{ff}$. Leipzig 1903.

2) Journal of comparative Neurology and Psychology vol. 14 p. 85-92. 1904.

3) American Journal of Physiology vol. 13 p. 351-357. 1905.

4) Bethe, Allgem. Anat. u. Physiol. des Nervensystems S. 82 u. 113. 1903 , 
des benutzten Nervenstücks annähernd die gleiche ist (37,1 und $34,6 \mathrm{~cm}$ pro Sekunde). Hieraus wird der Schluss gezogen, dass sich die leitende Substanz wie eine Flüssigkeit verhält. Unter dem Gesichtspunkt der oben gestellten Frage betrachtet würde sich ergeben, dass das Nervenfaserplasma das Leitende ist.

Ein Blick auf die Zahlen der einzeln aufgeführten Versuche zeigt aber, dass die Schlüsse der Autoren sich doch nicht so zwanglos ergeben, wie es nach ihrer Statistik erscheint. Die Zahlen schwanken hier für die gleichen oder annähernd gleichen Längen des ausgenutzten Stückes zwischen so weiten Grenzen, dass die Annahme einer Fehlerquelle naheliegt, welche die ganzen Resultate in Frage stellt. So findet man im gleichen Versuch als Übertragungszeit :

$$
\begin{aligned}
& \begin{array}{c}
\text { (aus Tabelle I von } \\
\text { Jenkins und }
\end{array}\left\{\begin{array}{cccc}
\text { bei } 4,5 \mathrm{~cm} & \text { Länge } & 0,094 \\
& 5,0 & &
\end{array} \quad 0,142 \text { (statt } 0,104\right. \text { ) } \\
& \text { Carls o n) } \quad " 10,5 \quad " \quad " \quad 0,244 \\
& \text { " } 11,5 \quad " \quad \text { " } 0,395 \text { (statt } 0,266 \text { ) }
\end{aligned}
$$

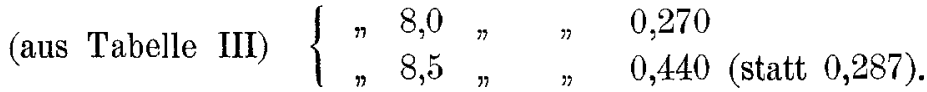

Die gefundenen Werte bei gestrecktem Nerv liegen manchmal unterhalb, meist aber oberhalb des theoretisch errechneten Wertes, im letzteren Falle häufig aber so weit von ibm entfernt, dass eine Übereinstimmung mit der Theorie nicht mehr anerkannt werden kann (s. Fig. $2 A$ ).

Die zweite Untersuchung wurde am Bauchmark eines Röhrenwurms (Bispira polymorpha) angestellt. Das Bauchmark wurde an zwei Stellen freigelegt (weit vorne und weit hinten) und gereizt. Die Reflexlatenz des Einziehens des Kopfes bei Reiz vorne wurde von der bei hinten erfolgendem Reiz abgezogen und so die Übertragungszeit gefunden. Das zwischenliegende Wurmstück wurde sich selbst überlassen oder gestreckt und so die Übertragungszeit bei verkürztem oder verlängertem Bauchmark gemessen. In diesen Versuchen ist zwar auch die Übertragungszeit bei gestrecktem Bauchmark stets grösser als bei verkürztem, sie erreicht aber in keinem Versuch eine der durchlaufenen Bauchmarksstrecke proportionale Länge, bleibt vielmehr in den meisten Versuchen weit unter diesem theoretischen Wert (s. Fig. $2 B$ ). Experimente, welche die Richtigkeit einer theorethischen Erwägung beweisen sollen, müssen aber Zahlen ergeben, welche um den theoretischen Wert herumliegen, sonst ist 
die Hypothese falsch. Mir scheint daher diese zweite Arbeit die Ansicht wenig zu unterstützen, dașs die leitende Substanz einen in seiner Länge variablen Flüssigkeitszylinder darstellt und im Nervenfaserplasma (Neuroplasma) zu suchen ist.

Eine eingehendere Kritik dieser Arbeiten ist erst möglich, nachdem die eigenen Untersuchungen besprochen sind.

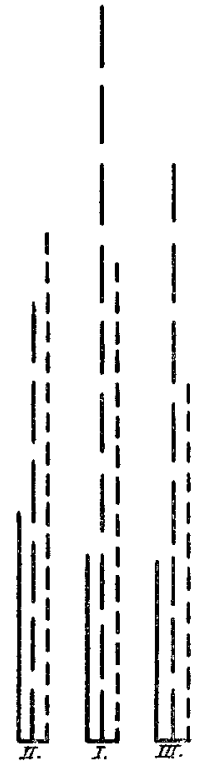

Fig. $2 A$.

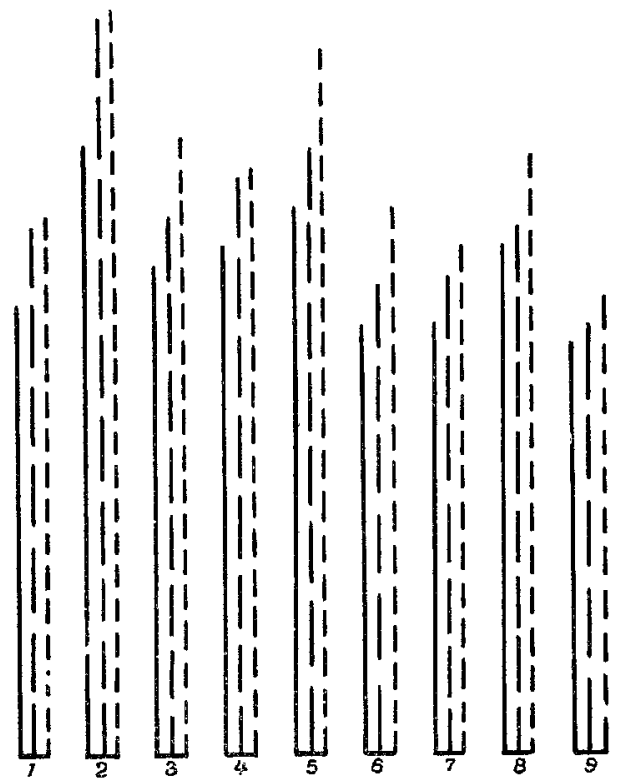

Fig. $2 \boldsymbol{B}$.

Fig.. 2. Graphische Darstellung der Resultate von Jenkins und Carls on an Ariolimax $(A)$ and von Carls on an Bispira $(B)$. Die erste ausgezogene Linie jeder Gruppe gibt die Länge der Übertragungszeit bei geringster Länge des Ubertragungsstückes an. Die zweite (langgestrichelte) Linie bedeutet die gefundene Länge der Übertragungszeit bei grösster Länge des Übertragungsstïckes. Die dritte (kurzgestrichelte) Linie zeigt, wie gross die Übertragungszeit bei grösster Länge des Übertragungsstückes sein müsste, wenn sie sich (nach der von den Autoren angenommenen Theorie) proportional der jeweiligen Länge des Nerven verhielte. $\mathrm{Zu} A$ : Als Unterlage konnten nur die drei in Extenso mitgeteilten Versuche (a. a. O. S. 89) dienen. Die erste Linie entspricht immer dem Wert der Übertragungszeit bei der kürzesten dort angegebenen Länge des Pedalnerven ( $I$. 1 , II. 2 und $I I I .2)$, die zweite Linie dem Wert bei grössester Länge $(I .8, I I .3$ und III. 3). Die Länge der dritten Linie wurde aus der Übertragungszeit bei kürzester Nervenlänge und dem Verhältnis der Nervenlängen berechnet. $\mathrm{Zu} B$ : die Länge der Linien wurde bestimmt aus der Tabelle II (a. a. O. S. 356) unter der Annahme, dass die Latenzzeit bei Reiz in der Nähe des Kopfes immer 0,021 Sekundèn betrug, welche Zahl für ein en Versuch (S. 354) angegeben wird. 


\section{Untersuchnngsobjekt und Kritik seiner Znlässigkeit.}

Als Versuchsobjekt diente mir der gewöhnliché Blutegel (Hirudo medicinalis). Es ist dies wohl das einzige zu derartigen Versuchen geeignete Tier des Binnenlandes. In bezug auf die Dehnbarkeit kann es auch den schönsten Objekten der marinen Fauna an die Seite gestellt werden. Ausserdem ist sowohl der Faserverlauf als auch das Verhalten der Neurofibrillen in den Nervenfasern bei diesem Tier besonders genau bearbeitet worden (Biedermann ${ }^{1}$ ), Apathy ${ }^{2}$ ), Bethe $\left.{ }^{3}\right)$ ].

Die peripheren Nerven der Hirudineen sind leider so kurz, dass sie für die vorliegende Frage nicht in Betracht kommen können. Dagegen scheint das Bauchmark, über dessen grosse Dehnbarkeit schon oben gesprochen worden ist, von vornherein geeignet. Nun ist aber das Bauchmark nicht ein einheitlicher Nervenstrang, sondern es ist zusammengesetzt aus Bündeln unverzweigter Nervenfasern, den Konnektiven, welche sich allein an der Dehnung beteiligen, und Ganglienknoten, in denen sich ein grosser Teil der Konnektivfasern verzweigt, und von denen in jedem Segment die peripheren Nerven erst abgehen. Da die Ganglien als Sitz der Reflexbogen aufzufassen sind, so kann es sich bei jeder Reaktion, die durch direkte oder indirekte Reizung des Bauchmarks hervorgerufen wird, immer nur um einen Reflex handeln. Die Untersuchung muss lehren, ob die Reflexzeit eine genügende Gleichmässigkeit besitzt, um Irrtümer in der Deutung der Resultate auszuschliessen.

Eine weitere Frage ist die, ob man die Bauchganglienkette für die vorliegende Frage als Nerv ansehen darf. Dies ist zunächst nur dann zulässig, wenn die Fasern, welche den Reiz vom entfernteren Reizpunkt demjenigen Ganglion zuleiten, dessen Reflexbewegung als Indikator benutzt wird, die dazwischen liegenden Ganglien ohne Unterbrechung passieren. Würde eine Reihe von zentralen Stationen (Faseraufsplitterungen, Zentren, Synapsen oder wie man sonst sagen mag) durchlaufen, so könnte eventuell die bei dieser Passage verloren gehende Zeit die Leitungszeit in den unverzweigten, dehnbaren Fasern der Konnektive überwiegen und kleine, zeitliche Unter-

1) Jenaische Zeitschr. f. Naturwissenschaft Bd. 25 S. 434 ff. 1891.

2) Mitteil. d. zool. Station zu Neapel Bd. 12 S. 495 ff. 1897.

3) Arch. f. mikrosk. Anat. Bd. 51 S. 402, 1898 u. Allgem. Anat. u. Physiol. S. $17,36,39.1903$. 
schiede verdecken. - Es sind nun bei Hirudo tatsächlich zahlreiche Fasern vorhanden, welche das ganze Bauchmark durchlaufen und nur Seitenzweige in den einzelnen Ganglien abgeben ${ }^{1}$ ). $\mathrm{Zu}$ diesen Fasern gehören die sensorischen Bündel Apáthy's's), die nach ihrem Verhalten an der Peripherie mit Sicherheit als rezeptorische Fasern anzusehen sind. Es lässt sich auch feststellen, dass die in diesen Fasern enthaltenen Neurofibrillen - und auf diese kommt es hier vornehmlich an - die Ganglien zum Teil glatt, zum Teil unter Abgabe von Seitenästchen passieren. Wir besitzen also eine den Hintersträngen der Wirbeltiere vergleichbare, das ganze Bauchmark durchziehende rezeptorische Bahn, welche den für das Experiment geforderten Bedinsungen entspricht. (Neben diesen langen rezeptorischen Bahnen sind noch andere lange [intrazentrale] Bahnen vorhanden. - Es gibt auch rezeptorische Fasern, die sensorischen Schläuche Apáthy's, welche ein geringeres, nur auf ein Ganglion beschränktes zentrales Ausbreitungsgebiet haben.)

Ein strenger Beweis, dass die vorhandenen langen rezeptorischen und intrazentralen Bahnen die Fernreflexe vermitteln, lässt sich nicht erbringen; es wird aber allgemein angenommen, dass dies so ist. Man wird von dieser für alle Tiere mit zentralisiertem Nervensystem gemachten einfachsten Annahme nur dann abgehen, wenn zwingende Gründe dafür vorliegen. Einen solchen zwingenden Grund werden vielleicht die Gegner der Fibrillenlehre in den Resultaten dieser Arbeit erblicken. Vorläufig befinde ich mich aber noch in Übereinstimmung mit der allgemeinen Anschauung, wenn ich - wegen der Existenz der langen durchlaufenden Babnen - das Bauchmark wirbelloser Tiere und speziell das von Hirudo zur Entscheidung der vorliegenden Frage für geeignet erkläre. Dieser Ansicht ist ja offenbar auch Carlson gewesen, wenn er an dem Wurm Bispira arbeitete.

1) Wegen technischer Schwierigkeiten kann man bei Hir u d o eine einzelne Faser nur durch einige wenige Ganglien hindurch verfolgen. Nach der ganzen Lage der Dinge ist aber nicht daran zu zweifeln, dass die durchlaufenden Fasern kontinuierlich das ganze Bauchmark durchziehen, wie dies bei manchen Wirbellosen (Homarus [A $\ \backslash \mathrm{e}]$, Carcinus [B ethe]) direkt unter dem Mikroskop an Präparaten des ganzen Bauchmarks festgestellt werden kạnn.

2) Mitteil. d. zool. Station zu Neapel Bd. 12 S. 567. 1897. 


\section{Methodik.}

Nach den Vorversuchen erwies sich die vollständige oder auch nur teilweise Freilegung des Bauchmarks als unzweckmässig. Bei vollständiger Freilegung geht die Erregbarkeit schnell verloren, und bei jeder Dehnung bleibt ein Dehnungsrückstand, so dass grosse Längendifferenzen nicht mehr erzielt werden können. Ausserdem kann man nicht mehr erkennen, in welchem Zustand sich die Zentren befinden, da der Indikator dafür - die gesamte Körpermuskulatur zum grössten Teil abgetrennt ist. Hemmungsvorgänge spielen aber bei allen Wirbellosen eine grosse Rolle, und durch Einmischung dieser werlen die Resultate ganz unklar. - Auch eine teilweise Freilegung zur direkten Anbringung der Elektroden erwies sich als unzweckmässig, weil der Zug bei künstlicher Dehnung resp. spontaner Verlängerung in unnatürlicher Weise an den Ganglien zerrt und hierdurch Leitungsverzögerungen hervorgerufen werden. Die direkte Reizung des freigelegten Bauchstranges wurde daher nur für gewisse Kontrollversuche benutzt.

Eine weitere, nach Möglichkeit zu vermeidende Fehlerquelle wurde in der Anwendung von $\mathrm{Z}$ wangsdehnungen gefunden. Werden die Tiere über ihre Maximallänge gedehnt, so tritt mehr oder weniger vollständige Reflexlosigkeit oder wenigstens eine Abschwächung und Verzögerung der Fernreflexe ein. Aber auch bei Zwangsdehnung, welche unterhalb der Maximallänge bleibt, zeigen sich häufig Verlängerungen der Übertragungszeit, welche fehlen, wenn die Tiere spontan dieselbe oder grössere Längen annehmen. Bei Zwangsdehnungen ist die Dehnung des Muskelschlauchs besouders anfangs stets ungleichmässig, so dass einzelne Teile überdehnt werden und hierdurch eine Zerrung der Ganglien zustande kommt. Es kann auch bei nichtmaximalen Zwangsdehnungen zu (meist vorübergehenden) lokalen Leitungsunterbrechungen im Bauchmark kommen.

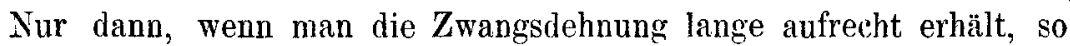
dass die anfangs stark tonisch kontrahierte Muskulatur erschlafft, treten wieder einigermaassen normale Leitungsverhältnisse ein.

Ich zog es daher vor, die Tiere selber ihre Länge variieren zu lassen. Dieses Verfahren stellt allerdings an die Geduld des Untersuchers ziemlich hohe Anforderungen, da die Tiere oft lange Zeit zwischen mittleren und starken Kontraktionszuständen hin- und herpendeln und die Maximallänge immer nur selten und dann nur 
vorübergehend einnehmen. Fhe man zu einer genügend grossen Anzahl von Bestimmungen kommt, können oft Stunden vergehen, und bei manchem Versuch wartet man überhaupt vergeblich auf geeignete Reizmomente für grössere Längen. Bisweilen kann man Verlängerung als Folgeerscheinung starker elektrischer Reizung oder durch sanftes Streichen des Rutckens mit einem nassen Pinsel erreichen. Zwangsdehnung wurdè am Ende des Versuchs dann angewandt, wenn auf andere Weise keine grossen Längen erzielt werden konnten.

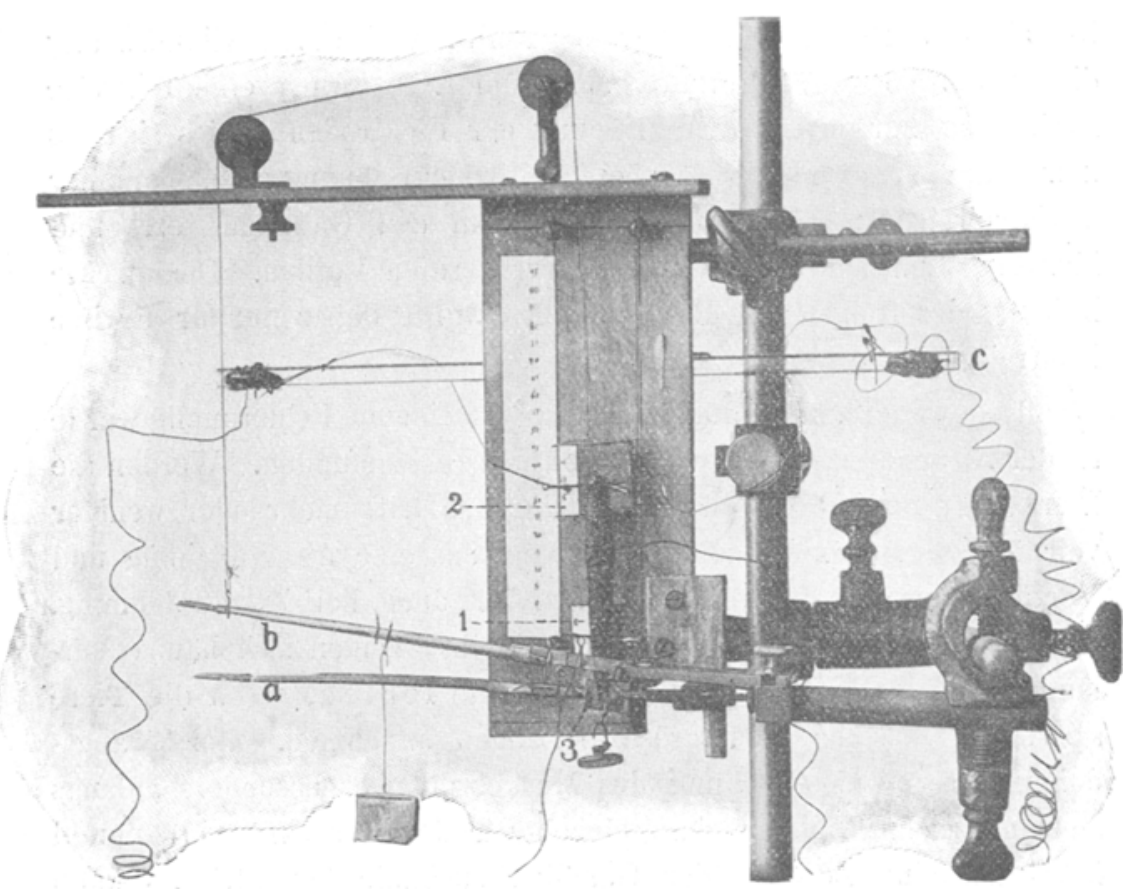

Fig. 3. Die zur Ermittlung der Übertragungszeit bei verschiedener Läzge des übertragenden Bauchmarkstückes benutzte Vorrichtung. $1=$ feste Korkplatte. $2=$ Korkschlitten mit Blechrahmen, welcher in Drähten geführt wird. $3=$ Korkplättchen am Hebel $\alpha$, welcher die zur Zeitmessung benutzten Kontraktionskurven des Vordertiers aufschreibt. $b=$ Hebel zur Markierung der jeweiligen Längen des Hintertiers (Übertragungsstück). $c=$ Glasstab zur Isolierung der Zuleitungsdrähte.

Um in dieser Weise arbeiten zu können, wurde folgende Einrichtung getroffen (Fig. 3): Auf einem Brett ist ein Korkstück (1) fest angebracht; auf welchem der Blutegel in der Gegend des Uterus, dessen Lage durch Palpation festgestellt werden kann, mit zwei Stecknadeln festgesteckt wird. Auf zwei in der Längs- 
richtưng. des Brettes gespannten 'Drähten: ist "verschieblich 'ein leichter Schlitten mit Korkplatte (2) angebracht." Auf diesen' wird das Hinterende des Tieres (dicht vor dem hinteren Saugnapf) mit zwei Nadeln festgespiesst. Von dem Schlitten geht ein dünner Faden aber zwei Rollen zur Spitze eines Schreibhebels (b), der mit 15-20 g belastet ist. Dièser Hebel dient nur dazu, um die jeweilige Länge des hinteren. Tierstücks, an dem die Ü̈bertragungszeit gemessen wẹrden soll; aus dem Kurvenblatt durch Umrechnung feststellen zu können. Neben dem Schlitten ist ein Maassstab auf dem Brett angebracht, so dass zur Kontrolle auch hier die Ablesung der Längen des Hịtertiers möglich ist.

Unter dem festen Kork ist ein zweiter Schreibhebel (a) angebracht. Dieser trägt ein Korkplättchen (3), auf welchem der: Kopf des Tieres mit einer Nadel befestigt wird. (Belastung unter dem Angriffspunkt, da Schleuderung bei der Langsanikeit der Bewegung kaum eine Rolle spielen kann und es ja auch nicht auf die Form der Kurve ankommt). Die Latenzzeiten des.vorderen, mit diesem. Hebel verbundenen Tierstücks (Vordertier) werden gemessen und zur Ermittelung der Übertragungszeit benutzt.

Als Stück von variabler Länge, in welchem' die 'Übertragungszeit festgestellt werden soll, dient das lange Hinterstück (Hintertier). Zur Reizzuleitung dienen die Nadeln, mit denen das Hintertier auf dem Schlitten (Schwanzreiz) und auf dem festen Kork (Kopfreiz) befestigt ist (an die Nadeln sind Lamettafäden resp: Dräbte angelötet).

Als $R$ e i z dienten entweder Öffnungsschläge - oder häufiger kurzdauernde. Reizperioden unter Einschaltung 'des Wagner'schen Hammers (45 Unterbrechungen in der Sekunde). Öffnungsschläge müssen in der Regel ziemlich kräftig sein, um sicher einen Reflex auszulösen. Es besteht daher die Gefahr, dass der Reiz. auf ein wesentlich grösseres Gebiet einwirkt, als man beabsichtigt. Bei Benutzung des Wagner'schen Hammers kann eine sichere. Wirkung bei wesentlich geringerem Rollenabstand erzielt werden. Es konnte durch Versuche festgestellt werden, dass bei eben sicher wirkender "tetanischer" Reizung (in der oben angegebenen Weise) 'der Reiz einen Aktionsradius von nicht mehr als $2-4 \mathrm{~mm}$ hat. (Wurde das Bauchmark 2-4 $\mathrm{mm}$ unterhalb der Reizstelle durchschnitten [gemessen bei mittlerem Kontraktionszustand]), so blieb das unterhalb 
gelegene Wurmstück beim Reizen in Ruhe.) - Der Nachteil der tetanisierenden Reize, dass man den Reizmoment nicht genau bestimmen könne, scheint mir nicht so gross, wie man meinen könnte. Besondere Versuche zeigten nämlich, dass bei ein und demselben Tier und bei gleichem Rollenabstand ein sicherer Reflex nach der g l eichen Unterbrechungszahl (2 resp. 3) eintrat. Da es ja nicht auf absolute Zahlen, sondern nur auf das relative Verhältnis der Übertragungszeiten ankam, so konnte die erste Unterbrechung als Reizmoment angenommen werden.

Da die Länge des reagierenden Stücks (des Vordertiers) nicht konstant ist, indem der Tonus dauernd schwankt, so müssen zur Ausmessung der Kurven die jeweiligen Fusspunkte der Kontraktionskurven mit Berücksichtigung der Bogenschreibung auf die Abszissenachse projiziert werden. Wegen der verschiedenen Anfangslänge ist es auch nicht möglich, Kontraktionen von gleicher Höhe zu erzielen.

Zur Zeitmarkierung reichte in der Regel der Jaquet'sche Chronograph aus. Durch Interpolation können die Latenzzeiten mit einer meist genügenden Genauigkeit von $0,01-0,02$ Sekunden bestimmt werden. Bei mehreren Versuchen wurde die Zeit mit einer Stimmgabel von 100 Schwingungen aufgeschrieben. Insbesondere gilt dies für die Versuche über Reflexzeit und Muskellatenz.

Die Tiere wurden auf dem kleinen Apparat stets in der natürlichen Lage befestigt, d. h. die Saugnäpfe der festen Unterlage zugewandt. Sie kommen, wenn sie sich festsaugen können, viel schneller zur Ruhe. Mit einiger Übung gelingt es, die normalen Tiere in die richtige Lage zu bringen; leichter ist es aber, wenn man die Tiere schwach mit Alkohol nach dem Vorgang von Fürst) narkotisiert. Die Tiere kamen für $2-3$ Stunden in $3-4 \%$ Alkohol, dann ebenso lange in $2 \%$ Alkohol. Sie sind dann noch sehr reaktionsfähig, aber ihre Bewegungen laufen sehr gleichmässig $a b$, und es fehlt ihnen die Kraft und die Sicherheit. Während des Versuchs wurde die Narkose durch Berieseln mit $2 \%$ Alkobol aufrecht erhalten, um Fehler zu vermeiden. Die Leitungsgeschwindigkeit ist nämlich auch bei schwach narkotisierten Tieren geringer als bei normalen. Bei stärkerer Narkose ist das Dekrement der Erregungsleitung sehr gross, so dass hierbei brauchbare Resultate nicht erzielt werden können.

1) Pflüger's Arch. Bd. 46 S. 370.1890. 


\section{Versuche.}

Ehe auf die Versuche zum eigentlichen Thema dieser Arbeit, nämlich auf das Verhalten der Übertragungszeit bei verschiedenem Dehnungszustand des Übertragungsstückes, eingegangen werden kann, sind eine Reihe von Vorversuchen zu besprechen, deren Kenntnis für die Kritik der Hauptversuche von wesentlicher Bedeutung ist. Diese Vorversuche beziehen sich auf das Verhältnis der Muskellatenz zur Reflexlatenz, auf die Abhängigkeit der Reflexzeit und der Latenzzeit der Muskeln von der Höhe des Muskeltonus und auf die zeitlichen Unterschiede zwischen Hemmungslatenz und Reflexlatenz.

\section{Reflexlatenz und Muzkellatenz.}

Wenn ein Blutegel in der angegebenen Weise einmal vorne und einmal hinten gereizt wird, so bedarf es noch der Prüfung, ob die gewonnenen Latenzzeiten (der Reaktion des Vordertiers) ohne weiteres voneinander abgezogen werden dürfen. Der Bewegungseffekt ist bei beiden Reizorten derselbe: das ganze Tier zieht sich mit Hilfe der Längsmuskeln zusammen. Bei Reiz hinten geht die Kontraktion von hier aus und ergreift immer weiter nach vorne gelegene Teile, wie man bei der Langsamkeit der Bewegung leicht mit freiem Auge feststellen kann; bei Reiz vorne (in der Gegend der Geschlechtsorgane) geht die Kontraktion vom Reizort nach beiden Enden hin. Es ist derselbe Effekt, den man am ungefesselten Tier beobachtet, wenn man es an verschiedenen Stellen mechanisch reizt.

Dass die Reaktion des Vordertiers bei Schwanzreiz (Reiz hinten) reflektorisch ist, steht ausser Frage, da die Reaktion ausbleibt, wenn die Bauchganglienkette zwischen dem Reizpunkt und dem Vordertier an irgendeiner Stelle (aber mindestens $2 \mathrm{~mm}$ vom Reizpunkt entfernt) unterbrochen wird. - Auch bei Kopfreiz (Reiz an der unbeweglichen Fesselungsstelle) muss es sich in der Hauptsache um einen Reflex handeln, da sich das Vordertier auch jetzt durch Kontraktion der Längsmuskeln verkürzt. Da nämlich die Stromfäden grösster Intensität senkreeht zum Verlauf der Längsmuskeln durch das Vordertier hindurch ziehen, so können sie diese nicht direkt erregen, wenigstens nicht in der angewandten Intensität ${ }^{1}$ ).

1) Siehe Straub, Pflüger's Arch. Bd. 79 S. 381. 1900. 
Bei dieser Anordnung der Elektroden werden aber die Zirkulärmuskeln der Länge nach durchströmt; dieselben könnten sich in der Nähe dèr Flektroden, die zu gleicher Zeit als Fesselung dienen, kontrahieren und hierdurch eine schwache an der Kurve sichtbare Hebung des Hebels bewirken. Die bei Kopfreiz geschriebene Kurve würde sich dann zusammensetzen aus einer kleinen Bewegung durch direkten Reiz der Zirkulärmuskeln und einer grösseren durch reflektorische Kontraktion der Längsmuskeln. Da nach Anạlogie der Verhältnisse bei andern Tieren zu vermuten wäre, dass die Muskellatenz bedeutend geringer sei als die Reflexlatenz, -so würde man bei Kopfreiz eine Muskellatenzzeit gewinnen und diese Zahl nicht von der bei Schwanzreiz gefundenen Reflexz eit abziehen dürfen.

Die zur Kontrolle gemachten Experimente zeigen, dass a u $\mathrm{ch}$ bei Kopfreiz die Reflexlatenz bestimmt wird. 1. Die mechanischen Verhältnisse sind für eine wirksame Übertragung der Kontraktion der Zirkulärmuskeln auf den Schreibhebel sehr ungünstig. Fine deutliche Hebung des Hebels tritt nach Herausnahne des Bauchmarks nur dann ein, wenn das Stück zwischen den Nàdeln gespannt wird, oder wenn sehr starke Reize angewandt werden. 2. Die zur Ermittelung der Übertragungszeit angewandten Reizstärken waren für die Zirkulärmuskulatur entweder unterschwellig oder so schwach, dass die Muskellatenz bei bester mechanischer Ausnutzung der Zugkräfte grösser als die Reflexlatenz wär.

Um dies festzustellen, wurden die Reiz- und Fesselungsnadeln rechts und links von einem Ganglion eingestochen. Oberhalb der Nadeln wurden die Konnektive zum nächsten Ganglion freigelegt, hochgehoben und mit Elektroden armiert, welche ebenso weit vom Ganglion entfernt waren wie die Nadeln. Reizung der Konnektive und Reizung in gewöhnlicher Weise wurde abwechselnd und mit yerschiedenen Reizstärken 'ausgeführt. Bei, anderen Reizungen wurde das ganze Vordertier der Länge nach durchströmt. Später wurde das Bauchmark herausgenommen und die Latenz der Längsmuskeln und der Zirkulärmuskeln an passenden, in der Verlaufsrichtung der Muskelfasern aufgespannten und durehströmten Streifen des Hautmuskelschlauchs bestimmt.

Um die gewonnenen Zahlen verwerten zu können, müssen die im folgenden Abschnitt mitgeteilten Befunde berücksichtigt werden: Es können nur solehe Zahlen 'miteinander' verglichen werden', bei 
deren Gewinnung der Tonus des reagierenden Tierstücks ungefähr derselbe war. Nach Herausnahme des Bauchmarks geht der Tonus immer stark herab und meistens unter den niedrigsten bei Gegenwart der Zentralorgane vorkommenden Tonusstand. Zur Publikation habe ich daher zwei Fälle ausgewählt, bei denen die Bestimmungen vor Herausnahme des Bauchmarks immer nur bei Tonustiefstand ausgeführt wurden und bei denen nach Herausnahme nur ein geringes weiteres Sinken des Tonus konstatierbar war.

Die Angaben der folgenden Tabellen sind Mittelwerte aus je drei bis fünf Bestimmungen.

\begin{tabular}{|c|c|c|c|c|c|c|}
\hline & $\begin{array}{l}\text { Rollen- } \\
\text { ab- } \\
\text { stand } \\
\text { in } \mathrm{cm}\end{array}$ & $\begin{array}{l}\text { Latenz bei } \\
\text { Reizung der } \\
\text { Konnektive } \\
\text { über dem } \\
\text { Ganglion } \\
\text { Sekunden }\end{array}$ & $\begin{array}{l}\text { Latenz bei } \\
\text { "Kopfreiz" } \\
\text { (Nadeln } \\
\text { rechts und } \\
\text { links vom } \\
\text { Ganglion im } \\
\text { Körper) } \\
\text { Sekunden }\end{array}$ & $\begin{array}{l}\text { Latenz der } \\
\text { Zirkulär- } \\
\text { muskeln } \\
\text { (ohne } \\
\text { Bauchmark) } \\
\text { Sekunden }\end{array}$ & \begin{tabular}{|c} 
Latenz \\
bei Längs- \\
durch- \\
strömung \\
des Vorder- \\
tiers \\
Sekunden
\end{tabular} & $\begin{array}{c}\text { Latenz der } \\
\text { Längs - } \\
\text { muskeln } \\
\text { (ohne } \\
\text { Bauchmark) } \\
\text { Sekunden }\end{array}$ \\
\hline I & $\begin{array}{c}12 \\
11 \\
10 \\
9,5 \\
9 \\
8 \\
7\end{array}$ & $\begin{array}{l}0,06 \\
\mathbf{0 , 0 6 4} \\
0,06 \\
\\
0,055 \\
0,058\end{array}$ & $\begin{array}{l}\text { - } \\
\overline{0,12} \\
0,09 \\
\mathbf{0 , 0 7} \\
0,06 \\
0,062\end{array}$ & $\begin{array}{l}\text { 二 } \\
\overline{-} \\
\overline{\overline{-}}, 095 \\
0,07 \\
0,068\end{array}$ & $\begin{array}{l}\overline{-} \\
\overline{-} \\
\mathbf{0 , 0 9 5} \\
0,06\end{array}$ & $\begin{array}{l}\overline{-} \\
\overline{-} \\
0,09 \\
0,057 \\
0,055\end{array}$ \\
\hline II & $\begin{array}{r}13 \\
12 \\
11 \\
10 \\
9 \\
8 \\
7 \\
6\end{array}$ & $\begin{array}{l}0,056 \\
0,065 \\
\mathbf{0 , 0 5 4} \\
\mathbf{0 , 0 5} \\
0,053\end{array}$ & $\begin{array}{l}\overline{-} \\
\overline{0,07} \\
0,056 \\
\mathbf{0 , 0 6 2} \\
\mathbf{0 , 0 5} \\
0,06 \\
0,055\end{array}$ & $\begin{array}{l}\bar{Z} \\
\overline{-} \\
\overline{0}, 075 \\
0,05 \\
0,047\end{array}$ & & \\
\hline
\end{tabular}

Die fettgedruckten Zablen sind von Kurven gewonnen, deren Steilheit und Höhe ungefähr gleich war. - bedeutet Unterschwelligkeit.

Bei maximalem Reiz sind die gefundenen Latenzen alle nahezu gleich gross, besonders im zweiten Versuch. Zwar sind Unterschiede zwischen der Latenz bei Konnektivreiz, bei Kopfreiz und Reiz des zentrenfreien Muskels (dessen Nerven aber noch vorbanden sind!) zu konstatieren, aber diese sind erstens nicht immer gleichsinnig und zweitens liegen sie noch nahezu innerhalb der Fehlergrenzen. Bei der geringen Anfangssteilheit der Kurven können nämlich nur noch 
die Hundertstel Sekunden einen Anspruch auf Genauigkeit machen ${ }^{1}$ ). Selbst wenn die Zirkulärmuskulatur bei Kopfreiz mitgereizt würde, so würde dies der Verwertbarkeit der gewonnenen Latenzzeiten keinen Abbruch tun. Da nun aber bei der Bestimmung der Übertragungszeit (siehe unten S. 26) stets schwache Reize (10-9 cm R.-A.) angewandt wurden und da bei solchen Reizen die Muskulatur noch gar nicht anspricht oder eine höhere Latenz als die Reflexlatenz besitzt, so ist die Latenz bei Kopfreiz als Reflexlatenz anzusehen und mit der Latenz bei Schwanzreiz vergleichbar.

Aus den gegebenen Zahlen lässt sich auch ein Grenzwert für die Grösse der Reflexzeit ermitteln:

Man wird vorläufig annehmen dürfen, dass es für den Reflex gleichgültig ist, ob der Reiz durch die Konnektive oder die peripheren rezeptorischen Fasern desselben Ganglions zugeleitet wird. Nun wirkte ein Reiz von 8-9 $\mathrm{cm}$ Rollenabstand, der $3-4 \mathrm{~mm}$ vom Ganglion entfernt direkt auf die Konnektive appliziert wurde (eine Entfernung, die der Länge der peripheren Bahnen ungefähr entspricht), noch nicht direkt erregend auf das Ganglion oder die von diesem ausgehenden Nerven ein (Kontrolle durch Abbinden). Bei solchen Reizen werden aber für die ganze Reflexlatenz im Durchschnitt 0,055 Sekunden gefunden. Die Durchschnittswerte der Muskellatenz bei maximalem Reiz unterscheiden sich von dieser Zahl nicht wesentlich, liegen sogar manchmal etwas höher. Man würde daraus den Schluss ziehen können, dass eine Verzögerung bei der Passage des Ganglions überhaupt nicht stattfindet, eine Möglichkeit, die nicht ausser acht $\mathrm{zu}$ lassen ist, da bei diesen Tieren ein direkter Zusammenbang der rezeptorischen und motorischen Bahnen nachgewiesen ist (Apáthy, Bethe, Prentis). Setzen wir aber den geringsten für Muskellatenz gefundenen Durchschnittswert von 0,047 Sekunden in Rechnung, so bekommen wir als Differenz der ganzen Reflexzeit und der Latenzzeit des Muskels einen Wert von 0,008 Sekunden $(0,055-0,047)$. Da in dieser Zahl noch die Leitungszeit in den Nervenstrecken mit mindestens 0,005 Sekunden enthalten. ist, so wird man die eigentliche Reflexzeit höchstens auf 0,003 Sekunden ansetzen dürfen.

1) Die Zeit wurde bei diesen Versuchen mit einer Stimmgabel von 100 Schwingungen in der Sekunde geschrieben. Geschwindigkeit der Trommelbewegung $60-70 \mathrm{~mm}$ in der Sekunde. 
Auf andere Weise würde sich die Reflexzeit vielleicht direkt bestimmen oder angeben lassen, ob bei diesen Tieren überhaupt ein Zeitverbrauch beim Passieren des Reflexbogens stattfindet. Solche Bestimmungen stossen aber bei der grossen Variationsbreite der Latenzzahlen und anderer ungünstiger Umstände auf grosse Sehwierigkeiten. Sie lagen auch nicht im Plan der Untersuchung. Der gefundene obere Grenzwert wird aber bei späteren Betrachtungen von Nutzen sein.

\section{Die Abhängigkeit der Latenzzeiten von der Höhe des Tonus.}

Der ganze Wurm hat keine bestimmte Ruhelänge. Bald verharren die Tiere stundenlang in maximal verkürztem Zustand, bald liegen sie für lange Zeit ganz- oder halbausgestreckt da. Schon die einfache Palpation überzeugt einen davon, dass die Muskulatur bei allen diesen Lagen schlaff sein $\mathrm{k}$ a $\mathrm{n} \mathbf{n}$, während sie sich bei den aktiven Bewegungen des Tieres (aber auch in manchen Dauerzuständen) und bei denselben absoluten Längen in starker Spannung befinden kann. Diese verschiedenen Zustände, bei denen sowohl die Länge wie die Spannung der Muskeln oft ganz unabhängig variiert, bezeichne ich mit v. U exküll ${ }^{1}$ ) als Tonuszustände ${ }^{2}$ ). Für das Tier

1) Zeitschr. f. Biolog. Bd. 44 S. 269, 1903 u. Bd. 46 S. 1.

2) Die Abhängigkeitsverhältnisse der Tonuszustände vom Zentralnervensystem sind durch v. Uexkülla) an einer Reihe wirbelloser Tiere untersucht worden, unter andern auch an Hirudob). Leider konnten seine Erfahrungen nur zum Teil für die vorliegende Untersuchung nutzbar gemacht werden, da bei derselben die Art der Fesselung neue Bedingungen schuf. In der Regel ähneln die Bewegungen, welche die Tiere in meinem Apparat ausführen, den von v. U exküll genauer analysierten Gehbewegungen. Ist hierbei die vollständige Kontraktion des ganzen Körpers eingetreten, welche infolge der Fesselung oft lange anhält, so ist ein Versuch nicht möglich, da das reagierende Vorderende maximal verkürzt ist. Dehnung des Vordertiers führt zu keinem Resultat, da die Muskulatur „gesperrt" ist. Erst, wenn ein neuer Schritt beginnt, streckt sich das Tier, indem die Streckung am Vorderende beginnt. Wenn die Übertragungszeit bei kurzem Übertragungsstück gemessen werden soll, so muss gereizt werden, ehe die Verlängerung auf das Hintertier übergeht. - Ist das Hintertier lang, so ist es in der Regel auch das Vordertier. Reizversuche sind dann leicht mit Erfolg anzustellen. Auf einen regelmässigen Wechsel von Verlängerungen und Ver-

a) Siehe Anmerinung 1.

b) Zeitschr. f. Biol. Bd. 46 S. 372. 1904. 
scheinen alle diese Zustände Ruhezustände sein zu können; ob sie das auch vom Standpunkt der Thermo-Dynamik sind, lässt sich zurzeit nicht entscheiden. Die Möglichkeit, dass es so ist, lässt sich jedenfalls nicht ausschliessen. Für die vorliegende Untersuchung spielt diese Frage auch keine Rolle; es kommt hier nur darauf an, dass die Muskulatur keine bestimmte Ruhelänge hat.

Schreibt man eine grössere Anzahl von Kurven des Vordertiers bei Kopfreiz auf, so variieren die gefundenen Latenzzeiten sehr erheblich. Die längsten Latenzzeiten können unter Umständen mehr als fünfmal so lang sein als die kürzesten. In diese Unterschiede der Latenzzeiten, die sich in derselben Weise auch bei direktem Reiz der Konnektive zeigten, lässt sich Ordnung hineinbringen, wenn man die Latenzzeiten nach den jeweiligen Längen des reagierenden Vordertiers ordnet. Die Latenzzeiten sind lang, wenn das Vordertier lang ist, $d$. h. der Tonus niedrig steht; die Latenzzeiten sind kurz, wenn das Vordertier kurz ist, der Muskeltonus also hoch ist. (Es scheint hierbei nur oder vorwiegend auf die Länge der Muskeln, die mehr oder weniger grosse Tonusmenge im Sinne v. Uexküll's, nicht aber auf die Spannung, den Tonusdruck im Sinne v. Uexküll's, anzukommen).

In den Figuren 4-6 sind drei Versuche von schwach narkotisierten Tieren dargestellt. Auf die Abszissenachse sind die jeweiligen Längen des Vordertiers abgetragen, als Ordinaten die dazu gehörigen Latenzzeiten. Die Punktreihen steigen mit unwesentlichen Abweichungen in schräggeneigter, meist etwas gekrümmter Linie auf. Unregelmässiger ordnen sich die Punkte bei nichtnarkotisierten Tieren. Ein Beispiel gibt die Fig. 7.

Auf verschieden günstigen Ablesungsbedingungen können diese Verschiedenheiten nicht beruhen, denn die niedrigsten Kurven (bei geringster, noch eine weitere Verkürzung zulassender Anfangslänge) gaben gerade die kürzesten Latenzzeiten und bei den höchsten und

kürzungen ist aber, wie schon bemerkt, nicht zu rechnen. - Lehrreich für die Abhängigkeit des Tonus sind die Versuche mit Zwangsdehnung; solange hierbei die Sperrung im gedehnten Hintertier andauert, bleibt das Vordertier verkürzt. Mit dem Nachlassen der Sperrung wird es länger und schliesslich ganz schlaff. Wird jetzt gereizt, so wird das Vordertier nach jeder Kontraktion gleich wieder schlaff. Wird die Dehnung aufgehoben, so tritt in der Regel langsame $\mathrm{Zu}$ sammenziehung des Hintertiers unter Sperrung der Muskulatur ein, welche das. Vordertier getreulich mitmacht. 
Ein neuer Beweis für die leitende Funktion der Neurofibrillen.

meist auch steilsten Kurven (bei mittlerer und grosser Anfangslänge) sind die Latenzzeiten am längsten. Die Ursache muss vielmehr in Eigenschaften des Präparates selber gesucht werden.

Mir scheint nun, dass es sich hier nicht um eine Eigenschaft des Nervensystems handelt, sondern um Bedingungen, die in der

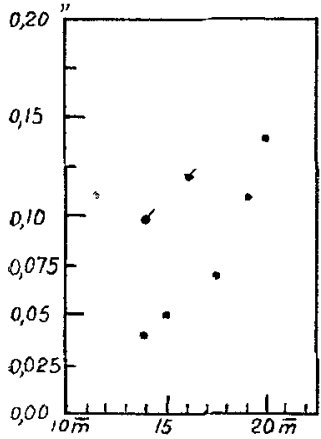

Fig. 4.

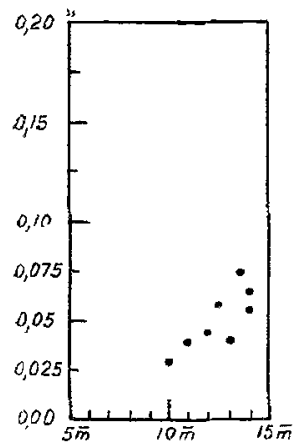

Fig. 6.

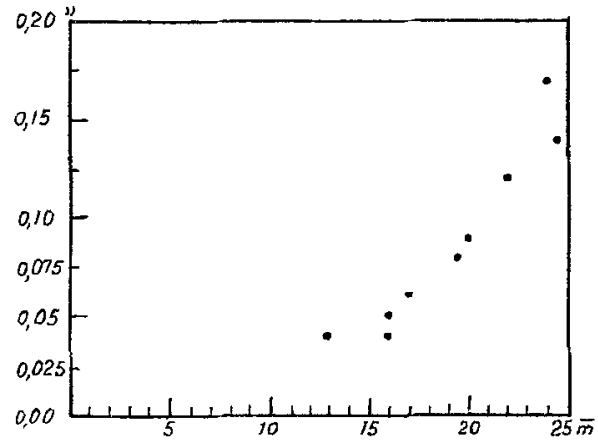

Fig. 5.

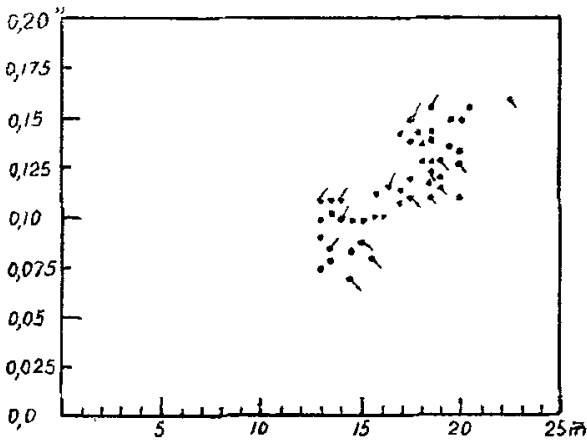

Fig. 7 .

Fig. 4-7. Latenzzeiten von Blutegeln (Vordertier bei Kopfreiz) geordnet nach den jeweiligen Längen des reagierenden Tierstücks. Die Längen des Vordertiers sind als Abszissen, die Latenzzeiten als Ordinaten eingetragen. Fig. 4-6 von schwach narkotisierten Tieren $(2 \%$ Alkohol), Fig. 7 von einem nicht narkotisierten Tier. Reizstärke bei Fig. $4=17 \mathrm{~cm} \mathrm{R.-A.} \mathrm{eines} \mathrm{grossen}$ $\mathrm{du} B$ o is. bei Fig. $5-7=10 \mathrm{~cm}$ Rollenabstand eines kleinen $\mathrm{du}$ Bois.

Muskulatur selber gelegen sind. Fntfernt man aus einem Stück Blutegel das Bauchmark, so sinkt der Tonus stark ab. Ansehnliche Tonussteigerungen wie beim Stück mit Bauchmark lassen sich nicht mehr erzielen. Immerhin kommen aber auch noch nach Entfernung des Zentralnervensystems Tonusschwankungen vor, welche in der Regel 5-10\%, bei manchen Präparaten aber bis zu $20 \%$ der 
grössten Länge ausmachen. An solchen Hautmuskelpäparaten mit grösseren Tonusschwankungen kann man bei genauer Ausmessung der Kurven ebenfalls konstatieren, dass die Latenzzeiten kurz sind, wenn die Anfangslänge gering ist, und länger sind, wenn die Anfangslänge gross ist. Die Figuren 8 und 9 geben Beispiele davon.

Würde man beim Muskel die Anfangslängen im selben Verhältnis variieren können, wie dies beim Tierstück mit erhaltenem Zentralnervensystem (bis zu 50\%) möglich ist, so würden die Unterschiede in der Latenzzeit voraussichtlich sehr viel ansehnlicher sein, sie würden aber bei proportionalem Anwachsen das Verbältnis bei diesen nicht erreichen. Man wird hierin keinen Grund für die An-

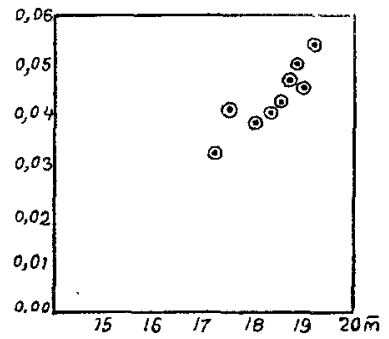

Fig. 8.

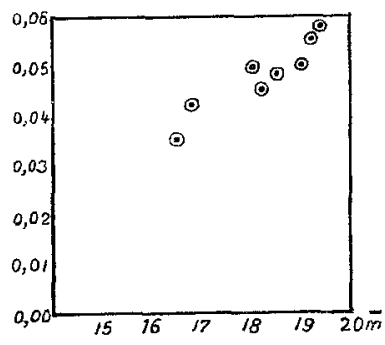

Fig. 9.

Fig. 8 und 9. Latenzzeiten von Muskelpräparaten von Blutegeln geordnet nach den jeweiligen Längen der Muskelstreifen. Die jeweiligen Längen des Muskelstreifens sind als Abszissen, die Latenzen als Ordinaten eingetragen. Reizstärke $7 \mathrm{~cm}$ Rollenabstand.

nahme erblicken dürfen, dass bei der Abhängigkeit der Latenzzeiten des normalen Tierstücks von der Anfangsiänge noch andere nicht in der Muskulatur gelegene Ursachen mitspielen. Es muss nämlich berücksichtigt werden, dass die Latenzen des normalen Tierstücks bei submaximalem Reiz, die der Muskelstreifen bei maximalem Reiz bestimmt wurden, bei welchem die Zeitdifferenzen verringert $\mathrm{zu}$ werden scheinen ${ }^{1}$ ). Ausserdem ist zu berücksichtigen, dass die $\mathrm{Zu}$ nahme der Latenzzeiten bei grösserer Ausgangslänge nicht geradlinig anzusteigen scheint.

1) Zur Reproduktion geeignete Latenzzeitbestimmungen vom unversehrten Tierstück bei maximalem Reiz habe ich bisher nicht bekommen und bin auch nicht auf deren Gewinnung besonders ausgegangen. Andrerseits gaben die Latenzzeitbestimmungen am Muskelstreifen bei submaximalem Reiz so verschiedene Werte, dass sie sich nicht gut ordnen liessen. 
Die Latenzzeiten des unversehrten Tierstücks scheinen ausser von seiner Länge auch von einigen anderen Faktoren abhängig zu sein, welche besonders bei nicht narkotisierten Tieren mehr in den Vordergrund treten. Genauer definieren kann ich nur einen dieser Faktoren: Ist die Anfangslänge, d. h. der augenblickliche Tonus bei der Reizung konstant, so lassen sich die Zeitwerte gut nach der jeweiligen Länge des Tierstücks ordnen. Ist aber der Tonus während der Reizung im Sinken begriffen (langsame Verlängerung des Tierstücks), so ist die Latenszeit in der Regel kürzer, als nach der Länge des Stücks zu erwarten war. Ebenfalls abweichend, aber nicht immer in einheitlichem Sinne, werden die Latenzzeiten gefunden, wenn der Tonus zur Zeit der Reizung im Steigen begriffen war. Meistens zeigen sich die Latenzzeiten in diesem Fall verlängert. In der Figur 5 und der Figur 7 ist bei den Reizpunkten, bei denen der Tonus in Veränderung begriffen war, die Richtung der Veränderung durch einen Strich angedeutet. Ein abschliessendes Urteil über diese Veränderungen der Latenzzeit und über ihre wirkliche Gesetzmässigkeit können erst weitere Untersuchungen bringen.

Für die Bestimmung der Länge der Übertragungszeit bei versehiedenen Längen ein und desselben Abschnittes des Bauchmarks spielt natürlich die Kenntnis dieser Verhältnisse eine wesentliche Rolle. Man darf nicht ohne weiteres die Latenzzeit bei Kopfreiz von der kurz darauf oder kurz vorher erhaltenen Latenzzeit bei Schwanzreiz abziehen, sondern darf nur zwei Latenzzeiten voneinander abziehen, die bei gleicher Lä nge des Kopfstücks bestimmt wurden. In den seltensten Fällen werden diese zeitlich und auf den Kurvenblättern nebeneinander liegen.

Hemmunglatenz und Reflexlatenz.

Bei Reizung des Blutegels in der beschriebenen Weise (S. 12) treten ausser Kontraktionen bisweilen auch Hemmungen ein. Natürlich können diese nur in Erscheinung treten, wenn das Tier (speziell das Vordertier) in mehr oder weniger verkürztem Zustand vom Reiz getroffen wird. Anstatt einer Zusammenziehung beobachtet man nach einer bestimmten Latenzzeit ein Nachlassen des Tonus, ein Absinken des Hebels. Die Erschlaffung kann sehr ausgiebig sein, sie kann bald zum Stillstand kommen, oder es kann kurz auf den 
Beginn des Absinkens eine Kontraktion folgen. In allerdings sehr seltenen Fällen bleibt das Absinken ganz aus, und es kommt nur zu einer sehr verspäteten Kontraktion. Alle diese Hemmungseffekte sind bei dem gewöhnlichen Versuchsverfahren („Kopfreiz" und "Schwanzreiz") selten, und ich habe sie bisher nicht willkürlich hervorrufen können; ich kann nur angeben, dass sich grosse individuelle Verschiedenheiten zeigen, und dass die Hemmungen bei schwächeren Reizen leichter eintreten als bei stärkeren. Man kann sich also in den Versuchen zur Ermittelung der Übertragungszeit fast ganz vor ihnen schützen, wenn man nicht zu schwache Reize benutzt.

Mit grösserer Sicherheit, aber auch nicht absolut sicher, erhält man die Hemmungseffekte bei Reiz der blossgelegten Konnektive. Bei eben wirksamen Reizen bekommt man sie bei manchen Exemplaren oft hintereinander, aber manchmal schlägt der Hemmungseffekt, auch ohne dass man den Reiz verstärkt hat, plötzlich ins Gegenteil um: es tritt Kontraktion ein.

Die Tatsache an und für sich wäre nicht weiter erwähnenswert, wenn nicht die Zeitverhältnisse an und für sich und im Zusammenhang mit den Resultaten von Jenkins und Carlson ein gewisses Interesse darböten. Bei Säugetieren treten nach den Versuchen von Sherrington ${ }^{1}$ ) die Hemmungseffekte mit einer geringeren oder mindestens ebenso kurzen Latenz in Erscheinung wie die Reflexkontraktionen. Das ist beim Blutegel nicht der Fall; die Hemmungslatenzen sind länger als die Latenzen der Reflexkontraktionen.

So wurde z. B. in einem Fall bei Reizung der Konnektive mit $10 \mathrm{~cm} \mathrm{R.-A.} \mathrm{gefunden:}$

Latenz der Reflexkontraktionen je nach

Länge des Vordertiers . . . . . . . 0,04-0,09 Sek.

Latenz der Hemmungseffekte bei starkemTonus

des Vordertiers . . . . . . . . . $0,17-0,18$ "

Latenz der Hemmungseffekte bei mittlerem

Tonus des Vordertiers . . . . . . 0,20-0,21"

Der Abfall der Kurve bei den Hemmungseffekten ist auch stets weniger steil als der Anstieg der Kurven bei Reflexkontraktionen.

Während die allgemeine Form der nervösen Vorgänge in der ganzen Tierreihe eine ziemliche Übereinstimmung zeigt, regelt sich

1) Proceed. royal Society vol. B. 76 p. 271. 1905. 
ihre spezielle Ausführung nach den Bedürfnissen des nervösen Betriebes der einzelnen Tierformen.
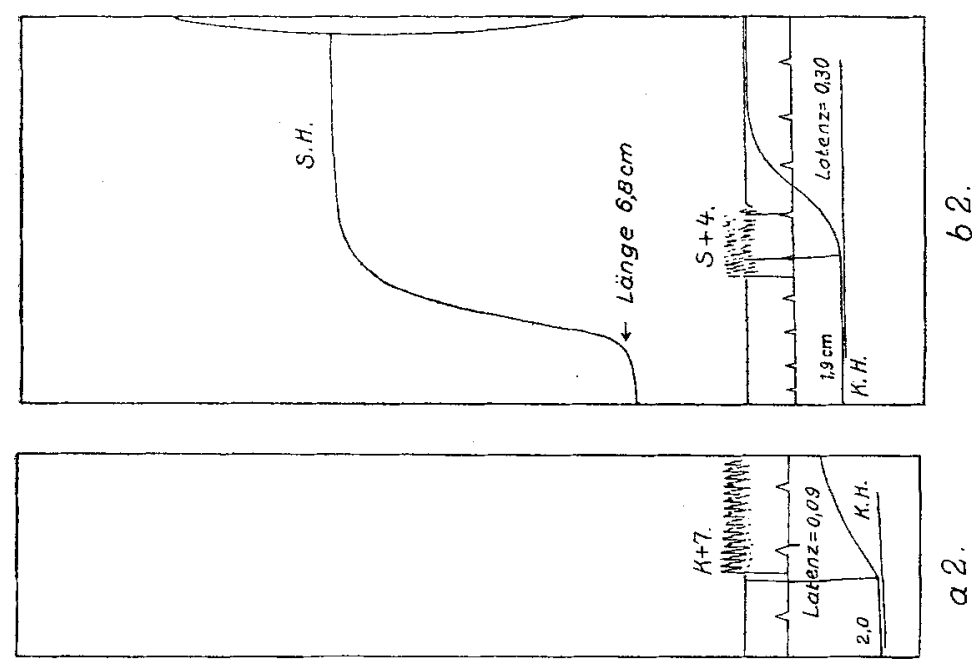

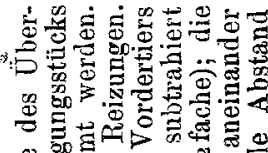

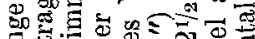

o :

-

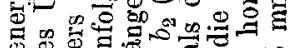
क्ष马 .

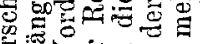

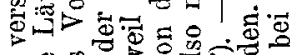

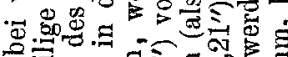
○击

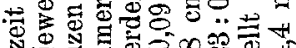

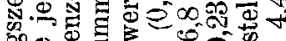

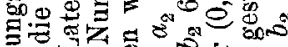

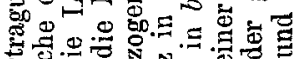
可歌

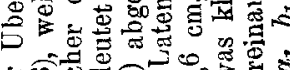
की की क्षे $\Leftrightarrow=20$

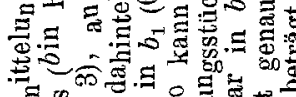

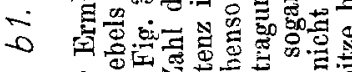

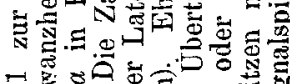

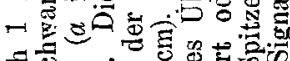

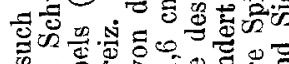

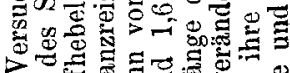

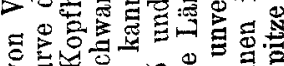

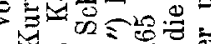
.

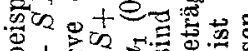

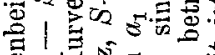

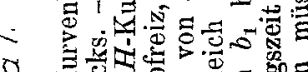

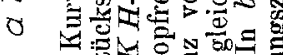
की @ 政

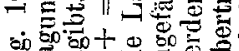

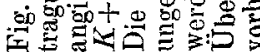

Von den Versuchen zur Bestimmung der Übertragungszeit werden im folgenden nur solche mitgeteilt, bei denen im Verlauf des Versuches Hemmungseffekte bei Reizung nicht zur Beobachtung kamen. 
Bestimmungen der Übertragungseit bei verschiedener Länge desselben Bauchmarkabschnittes.

Nachdem das Tier in der angegebenen Weise gefesselt war, wurde meist abwechselnd und $\mathrm{zu}$ geeigneten Momenten ein Schwanzreiz und ein Kopfreiz angesetzt. Es wurde darauf geachtet, nicht nur den Schwanzreiz bei möglichst verschiedenen Längen des Übertragungsstücks zu applizieren, sondern auch den Kopfreiz bei möglichst verschiedener Länge des Vordertiers. Über das Aussehen derartiger Kurven gibt die Fig. 10 Aufschluss.

Nach Berechnung der Latenzzeiten und der jeweiligen Längen des Übertragungsstücks und des Vordertiers wurden zunächst die Latenzzeiten bei Kopfreiz nach den jeweiligen Längen des Vordertiers geordnet. Die Durchschnittswerte der zu bestimmten Längen gehörigen Latenzzeiten wurden in einem Koordinatensystem als negative Werte auf der Ordinatenachse abgetragen und durch diese Punkte parallel zur Abszissenachse verlaufende Linien gelegt.

Auf der in Zentimeter geteilten Abszissenachse wurde die Länge des Ü̉bertragungsstücks bei grösster Verkürzung als Länge $a$ abgetragen. Die dazugehörige Latenzzeit (bei Schwanzreiz) wurde als Ordinate von dem entsprechenden Punkt derjenigen zur Abszisse parallelen Linie eingetragen, welche der Latenzzeit des Vordertiers bei „Kopfreiz" und gleicher Länge des Vordertiers zukam. In derselben Weise wurden die bei anderen Längen des Übertragungsstücks und ebenfalls bei "Schwanzreiz" gefundenen Latenzzeiten eingetragen. Da die Punkte unter der Abszisse, von welchen aus nach oben abgetragen wurde, (mit dem gleichen Zeichen) markiert wurden, übersieht man in den beigegebenen Fig. 11-14, welche Abzüge von der Latenz bei Schwanzreiz zur Ermittelung der Übertragungszeit gemacht wurden.

Eine gestrichelte Linie vom Nullpunkt durch den Punkt bei geringster Länge des Übertragungsstücks zeigt an, in welcher Weise sich die Übertragungszeit ändern müsste, wenn sie sich proportional der jeweiligen Länge des Übertragungsstücks verhielte. Kreuze auf dieser Linie deuten an, wie gross in diesem Fall die Übertragungszeit an der Stelle der zugehörigen Ordinate zu sein hätte.

In den vier abgebildeten Versuchen liegen alle Punkte nicht auf dieser Linie, sondern auf einer der Abszissenachse nahezu parallelen Linie. Die Übertragungszeit bleibtalso bei ver schiedenen Längen des Übertragungsstücks konstant. 


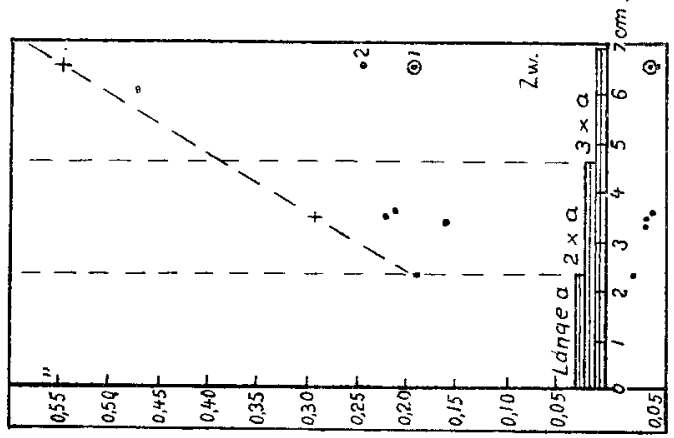

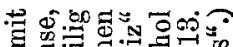
응

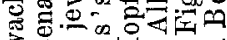

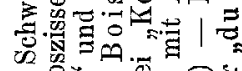
- $=8 \% 3$

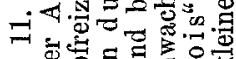

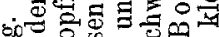

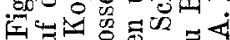
ह $=$ का

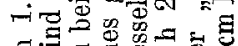

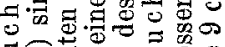

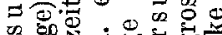
क

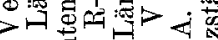

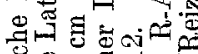

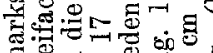

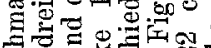
曲焉 ब

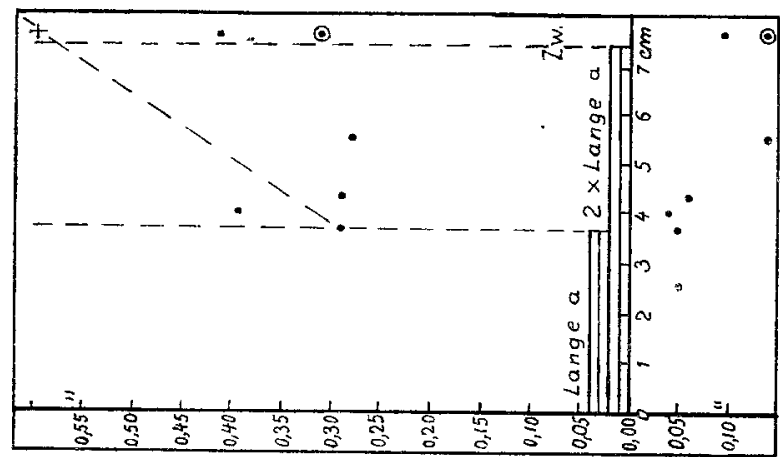
$0 \%$

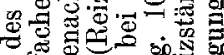

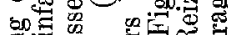

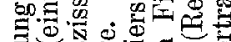
范宓

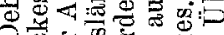
ब

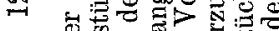
i

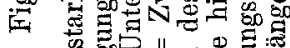

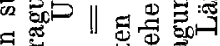

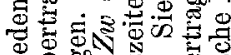
政

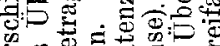

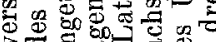
$>$. E ๑ี

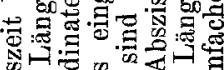

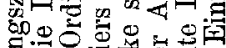

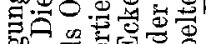

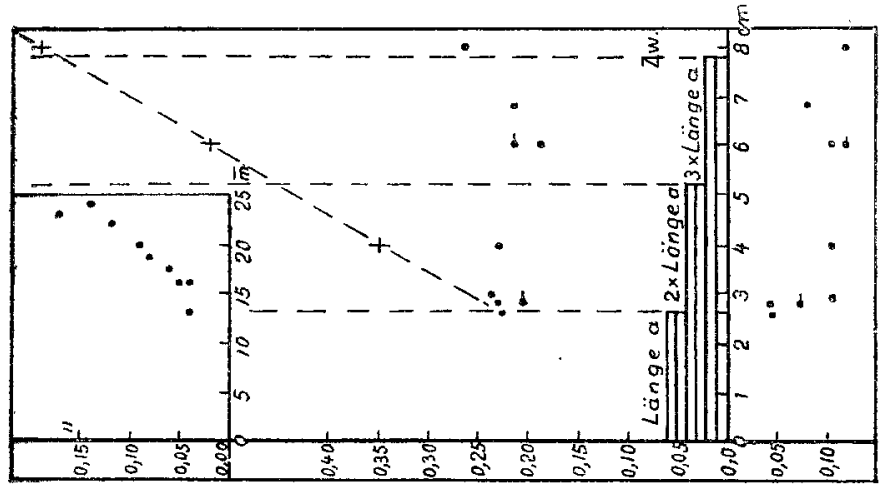

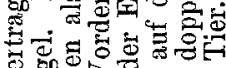
: 5 웝 空 六 它包

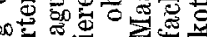

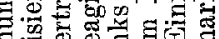

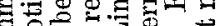

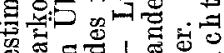
苟哥

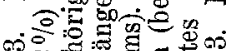

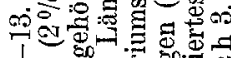
-

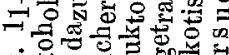
s. 
In dem Versuch 1 (Fig. 11) sind die bei der mehr als doppelten Länge $a$ gefundenen Übertragungszeiten etwas (aber wohl noch innerhalb der Fehlergrenzen) kleiner als bei einfacher Länge. Nur der bei Zwangslänge von mehr als der dreifachen Anfangslänge gefundene Punkt liegt etwas über der Parallelen, erreicht aber nicht

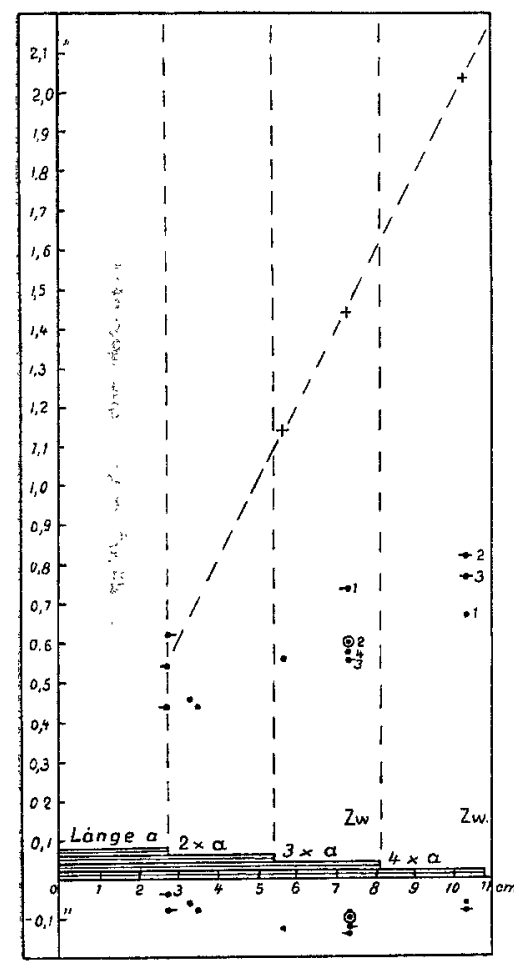

Fig. 14. Bestimmung der Übertragungszeit bei einfacher bis vierfacher Länge des Übertragungsstückes. Versuch 4 . Schwach mit Alkohol narkotisierter Blutegel. $Z_{w}=Z_{\text {wangslängen. Die }}$ Zahlen bei den Latenzzeitpunkten geben die Reihenfolge der Reizungen bei den betreffenden Zwangslängen an. (Reizstärke $9 \mathrm{~cm} \mathrm{R}$.-A. kleiner du B o i s). im entferntesten den Wert, den er bei proportionaler Übertragungszeit resp. bei (in bezug auf die Nervenlänge) konstanter Leitungsgeschwindigkeit haben müsste. In Versuch 2 und 3 ist ein ähnliches Verhalten zu bemerken. Der schlechte Punkt bei doppelter Länge in Versuch 2 (Fig. 12) wird durch den ebenfalls hoch über den anderen liegenden Punkt bei fast einfacher Länge aufgewogen.

In Versuch 4 (Fig. 14) wurde Zwangsdehnung über die natürliche Maximallänge versucht. Die gefundenen drei Punkte liegen alle oberhalb einer durch den Punkt geringster Länge gezogenen Parallelen, der unterste allerdings noch beinahe innerhalb der Fehlergrenzen. Von den vier bei geringerer $Z_{\text {wangslänge gewon- }}$ nenen Punkten liegen drei ungefähr auf der Parallelen, der vierte wesentlich dariuber. Es ist dies der zuerst gewonnene, und es zeigt sich hier das schon (S. 11) erwähnte Verhalten, dass bei

Zwangslänge auch unterhalb der physiologischen Maximallänge eine Verzögerung der Leitung zu konstatieren ist, so lange die Muskeln gesperrt sind. Erst wenn die Muskulatur erschlafft und dadurch die lokalen Zerrungen an den Ganglien aufhören, treten wieder normale Leitungsverhältnisse ein; die Punkte liegen dann auf der Parallelen. 
Der Versuch 5 (Fig. 15) ist nach einem anderen Prinzip angestellt. Es wurden nur die Latenzzeiten bei Schwanzreiz bestimmt und diese im unteren Teil der Fig. 15 nach Längen des Hintertiers (Übertragungsstück) geordnet. Sie liegen auf

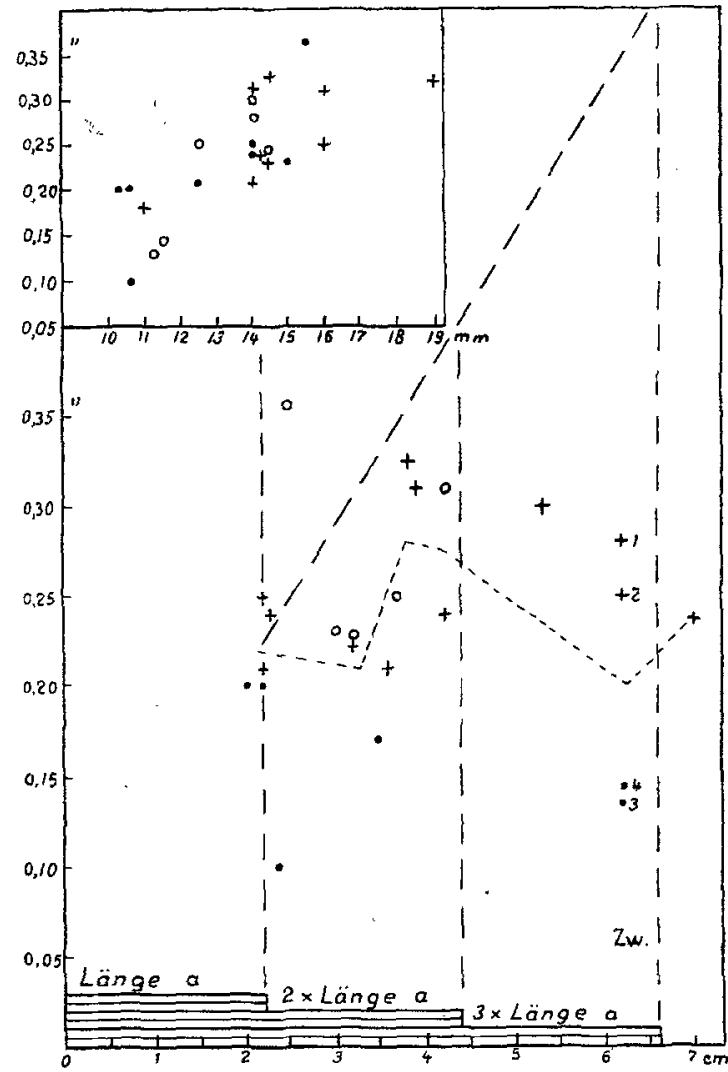

Fig. 15. Versuch 5. Latenzzeiten bei Schwanzreiz bei einfacher bis dreifacher Länge des Übertragungsstïckes. Nicht narkotisiertes Tier (Reizstärke $9 \mathrm{~cm}$ R.-A. kleiner $\mathrm{du}$ B o is). Im unteren Teil sind die Latenzzeiten nach Längen des Übertragungsstückes geordnet. Die Latenzzeiten bei 10-12 mm Länge des reagierenden Vordertiers sind im unteren Teil der Figur mit schwarzen Punkten markiert (๑). Die Latenzzeiten bei $12,1-14,9 \mathrm{~mm}$ Länge des Vordertiers mit Kreuzen $(+)$, bei $15-19 \mathrm{~mm}$ mit kleinen Kreisen $(0)$. $Z w=Z$ wangslänge. Die Zablen bei den Punkten geben die Reihenfolge der Reizungen an. Im oberen Teil der Figur sind dieselben Latenzzeiten geordnet nach der jeweiligen Länge des reagierenden Vordertiers. Hi er bedeutet ein Punkt (•) eine Länge des Ü bertragungsstücks von $20-30 \mathrm{~mm}$, ein Kreuz $(+)$ eine Länge des Übertragungsstücks von $81-50 \mathrm{~mm}$ und ein Kreis $(O)$ eine Länge desselben von $51-70 \mathrm{~mm}$.

einem ziemlich breiten, etwa horizontal verlaufenden Streifen zerstreut. Die aus einem senkrechten Flächenstreifen von je $5 \mathrm{~mm}$ 
Breite gezogene Durchschnittslatenz ergibt die zackige, kleingestrichelte Linie, welche in ihrer Hauptrichtung horizontal verläuft. In der Figur sind die Latenzzeiten je nach der Länge des Vor dertiers in drei Gruppen (mit einheitlicher Markierung) geteilt, wobei sich zeigt, dass die Latenzen bei geringster Länge des Vordertiers, gleichgültig, wie lang das Übertragungsstück war, am kürzesten sind. Links oben in der Figur sind dieselben Latenzen $n$ ach der Länge des Vordertiers geordnet. Sie ergeben hier einen ziemlich gleich breiten, schräg aufsteigenden Streifen, in welchem die gleichbezeichneten Punkte (bei ungefähr gleicher Länge des Übertragungsstücks) bunt durcheinander liegen. Der Streifen ähnelt dem in Fig. 7 abgebildeten, durch gleichartige Ordnung der Latenzen bei Kopfreiz gewonnenen.

Das Resultat ist hier auch wieder dasselbe wie bei den vorigen vier Versuchen: Die Übertragungszeit bleibt bei verschiedener Länge des Übertragungsstücks gleich. Die Unterschiede in der Latenz bei Schwanzreiz erklären sich aus der Verschiedenheit der Länge des Vordertiers (s. vorigen Abschnitt).

Ich glaube noch besonders hervorheben zu sollen, dass die wiedergegebenen Versuche nicht ausgewählte Beispiele sind, bei denen es sich zufällig gerade so getroffen bat ${ }^{1}$ ). Diesen Versuchen stehen keine anderen gegenüber, in denen das Resultat prinzipiell anders ausgefallen wäre. Wenn ich keine weiteren Versuche wiedergebe, so liegt dies daran, dass in den übrigen Versuchen, die ich angestellt habe, die Zahl der Reizungen entweder zu gering ist, um ein deutliches Bild zu geben, oder nur Reizungen bei geringen Längendifferenzen des Übertragungsstücks (aus schon oben angegebenen Gründen) erzielt werden konnten. Auch diese nicht mitgeteilten Versuche führten zu prinzipiell dem gleichen Resultat.

\section{Kritik der Versuche von Jenkins und Carls on.}

Die meinen Resultaten durchaus entgegengesetzten Befunde von Jenkins und Carlson, die schon oben besprochen wurden, lassen es wünschenswert erscheinen, den Gründen der Verschiedenheit nachzuforschen. Da ich die Versuche nicht mit angesehen habe und die Versuchsanordnung dieser Autoren nur kurz angedeutet wird, so bin ich auf Vermutungen angewiesen, glaube aber doch dieselben bei der Wichtigkeit der Frage mitteilen zu sollen.'

1) In die beigegebenen $\dot{K} u r v e n$ wurden alle in jedem der Versuche gefundenen Werte der Übertragungszeit eingetragen. 
Dass die wirklichen Befunde von Jenkins und Carlson mit ihren ${ }_{\mathbf{t}}$ heoretischen Schlüssen nicht in dem wünschenswerten Einklang stehen, habe ich schon am Anfang anseinandergesetzt (S. 6). Die an und für sich unwahrscheinl iche Annahme, dass bei einem Tier (Hirudo) die Übertragungszeit konstant ist, bei anderen (Ariolimax, Bispira) aber proportional der Dehnung ist, kann deshalb ausser Acht gelassen werden.

$Z$ war sind die Fibrillenverhältnisse bei den erwähnten Objekten nicht untersucht, aber ich glaube doch annehmen zu dürfen, dass anch hier die Fibrillen so lang sind wie die maximale, physiologische Nervenlänge. Ein Vergleich mit meinem Objekt wäre danach zulässig. Dass nun in allen Versuchen die Maximallänge innegehalten wurde, dafür finde ich keine Beweise. Es könnte daher eine Fehlerquelle darin bestehen, dass die Nerven bei einigen Versuchen überdehnt wurden, wodurch wie gezeigt sehr starke Leitungsverzögerungen zustande kommen können. - Bei beiden Versuchsreihen wurde am freipräparierten Nerven resp. Konnektiv gearbeitet. Nur bei Bispira wurden lokale Dehnungen vermieden, bei Ariolimax nicht. In ersten Fall waren die Übertragungszeiten meist we nig vergrössert, im letzteren sehr stark, oft sogar weit über den theoretischen Betrag hinaus (bei Annahme einer proportionalen Zunahme).

Bei Pulmonaten sind die Muskellängen noch weniger konstant als bei Würmern. In den genauer mitgeteilten Versuchen an Ariolimax (einem Pulmonaten) schwanken die Latenzzeiten bei Reiz dicht am Muskel in sehr weiten Grenzen. Es ist daher zu vermuten, dass auch hier die Latenz von der jeweiligen Länge abhängig ist (Siehe oben S. 19). Dies war den Autoren wohl nicht bekannt und daher wurden immer zwei nacheinander gewonnene Latenzzeiten ohne Räcksicht auf die Länge des reagierenden Muskels voneinander abgezogen, wodurch natürlich bedeutende Fehler entstehen können,

In den diesen beiden Arbeiten beigegebenen Kurvenbeispielen liegt die Kontraktionskurve trotz der wechselnden Länge des Muskels stets in gleicher Höhe äber der Signallinie. Wie aus einer anderen Arbeit derselben Autoren ${ }^{1}$ ) zu erseben ist, haben dieselben, um die grossen Längenverschiedenheiten des reagierenden Muskelstücks auszugleichen, das ganze Präparat im Verhältnis zum Hebel bewegt. Bei sehr guter Parallelführung und genauer Einstellung auf ein stets gleiches Niveau ist theoretisch hiergegen zwar nichts einzuwenden. Da solche Präparate aber, wenigstens nach meinen Erfahrungen, häufig sehr schnell ihre Längen wechseln, so dürfte eine genaue Einstellung auf das gleiche Niveau nicht immer möglich sein; bei senkrechter Projektion auf die Zeitschreibung, wie sie stets vorgenommen zu sein scheint, müssen dann Fehler entstehen, deren Grösse von der Länge des Hebels abhängig ist.

Schliesslich wird das Ariolimaxpräparat von Jenkins und Carlson ohne weiteres als Nervmuskelpräparat angesehen. Das ist es aber wohl sicher nicht. Schon bei Würmern treten, wie oben erwähnt, auf Reiz der Konnektive leicht Hemmungserscheinungen zutage, die sich mit nachfolgenden Kontraktionen kombinieren können. Das anfängliche Sinken des Tonus kann sogar ausbleiben und nur eine wesentlich verspätete Kontraktion in Erscheinung treten. Sehr viel 
häufiger sind solche Hemmungserscheinungen bei Reizung der Pedalnerven von Mollusken, welche nicht nur von mir, sondern auch von Biedermann und einer Reihe von Zoologen aus anatomischen und physiologischen Gründen als Konnektive angesehen werden. Reizung der Pedalnerven bei Aplysia, Limax, Arion [Bethe $\left.\left.\mathrm{e}^{1}\right)\right]$ und Helix [Biedermann $\left.{ }^{2}\right)$ ] bewirkt meistens auch bei ziemlich bedentenden Reizstärken einen Wechsel von Kontraktionen und Erschlaffungen. Das Primäre ist häufig (besonders bei Helix und Aplysia) eine Erschlaffung. Wenngleich die Autoren derartige Effekte bei Ariolimax nicht erwähnen, so glaube ich doch, das Vorkommen derselben auch hier vermuten zu dürfen. Es liegt nicht ausser dem Bereich der Möglichkeit, dass die Dehnung der Pedalnerven die Hemmungseffekte besonders bei muskelferner Reizung begünstigt. Da hierbei die Muskeln meist sehr niedrigen Tonus haben werden, so werden sich Hemmungseffekte nur in verspäteter Kontraktion äussern können. Jedenfalls wird man nach den bisherigen Erfahrungen an Mollusken nicht ohne Rücksicht auf diese Verhältnisse arbeiten und diese Tiere überhaupt nicht als besonders geeignetes Objekt für den vorliegenden Zweck ansehen dürfen.

Durch alle diese Fehlerquellen würde sich sowohl die Inkonstanz der Resultate von $\mathrm{J}$ enkins und $\mathrm{Carls}$ on als auch ihr scheinbar prinzipieller Gegensatz zu den Ergebnissen meiner Versuche erklären lassen.

\section{Schlussbetrachtungen.}

Nach den oben mitgeteilten Versuchen kann die Länge einer gegebenen Bauchmarkstrecke auf das doppelte und dreifache (das ist innerhalb der physiologisehen Grenzen) gedehnt werden, ohne dass die Übertragungszeit in merklicher Weise verändert wird. Erst bei Dehnung über die physiologische Länge tritt eine Verlängerung der Übertragungszeit ein. Die Geschwindigkeit der Reizleitung in einem gegebenen Stück Bauchmark ist also proportional seiner augenblicklichen Länge.

Hieraus ergibt sich als Antwort auf die am Anfaug aufgeworfene Fragestellung: Das leitende Element oder besser gesagt, das die Leitungsgeschwindigkeit bestimmende Element des Nervensystems sind die Neurofibrillen. Denn die Neurofibrillen bewahren innerhalb der physiologischen Längendifferenzen der Nerven die gleichen Längen, so dass eine Verlängerung des Nerven innerhalb dieser Grenzen an ihrer Länge und der Länge der Übertragungszeit nichts ändern kann. Erst wenn die Dehnung des Nerven über die natürliche Maximallänge hinausgeht, werden

1) Allgem. Anat. u. Physiol. d. Nervensystems S. 113-121. 1903.

2) Pflüger's Arch. Bd. 107 S. 48 u. 49.1905. 
auch die Fibrillen gedehnt, und nun nimmt auch die Ubertragungszeit zu.

Dieser Sehluss hat zunächst seine Berechtigung unter der Annahme, dass die langen rezeptorischen Bahnen, welche das ganze Bauchmark durchziehen, wirklich der direkten Reizleitung von einem Punkt zu einem entfernten Reizort dienen. Aber auch dann, wenn man diese Annahme nicht macht, sondern der Ansicht ist, dass die Reizleitung durch kurze Bahnen von Ganglion zu Ganglion vermittelt wird, - auch dann bleiben die mitgeteilten Versuche beweiskräftig. Es konnte festgestellt werden (S. 18), dass der obere Grenzwert für die Dauer der Reflexzeit 0,003 Sekunden beträgt. Zwischen hinterem Reizort und Reflexort lagen bei den Versuchen 11-12 Ganglien ${ }^{\mathfrak{y}}$ ). Wenn wir den maximalen, aber sicher viel zu hoch bemessenen Wert für die Reflexzeit in Rechnung setzen, so würde auf dem Wege durch die Ganglien eine Verzögerung von 0,033 bis 0,036 Sekunden stattfinden. Es bliebe also z. B. in Versuch 1 bei einer geringsten Länge des Übertragungsstückes von $23 \mathrm{~mm}$ und einer Übertragungszeit von 0,22 Sekunden für die Leitung in den Konnektiven eine Zeit von ca. 0,184 Sekunden. Bei doppelter Länge des Übertragungsstückes und gleichbleibender Leitungsgeschwindigkeit müsste eine Übertragungszeit von $0,22+0,184=0,404$ Sekunden gefunden werden. Die tatsächlich gefundenen Werte liegen aber nicht über dem Anfangswert von 0,22 Sekunden.

Also auch dann, wenn man die unwahrscheinliche Annahme macht, dass die langen Bahnen der Fernleitung nicht dienen, dass, Rer Reiz vielmehr in jedem Ganglion Station macht, bleibt der Schluss berechtigt, dass die Neurofibrillen die Leitungsgeschwindigkeit bestimmen.

In keiner Weise lassen sich meine Befunde nach den bisherigen Annahmen über die Nervenleitung dahin deuten, dass das Nervenfaserplasma (die Perifibrillärsubstanz) das Leitende ist. Da dieses nachweisbar bei den Längenveränderungen des Nerven seine Länge verändert, so müsste die Übertragungszeit proportional der jeweiligen Länge sein, was nicht zutrifft. Nur durch sehr gezwungene Hilfshypothesen würde man die Konstanz der Übertragungszeit mit der

1) Von den 21 Ganglien liegen im Übertragungsstück nưr das 7. bis 17. (resp. 18.) Das 6. Ganglion ist das eigentlich reagierende. Die vordersten und hintersten Ganglien haben nur geringe Abstände und wurden deshalb ausgeschlossen. 
Ansicht von der leitenden Funktion des Nervenfaserplasmas in Finklang bringen können.

Wenn die Fibrille die Leitungsgeschwindigkeit bestimmt und nicht das Nervenfaserplasma, so geht daraus noch nicht hervor, dass die Fibrille die Leitung ohne Mitbeteiligung anderer Elemente des Nerven besorgt. Es erhellt nur, dass die Neurofibrillen die Hauptträger der Erregungsleitung sind. Sie könnten die Leitung allein besorgen, sie könnten aber auch beim Leitungsprozess in Wechselwirkung mit anderen Elementen der Umgebung stehen. So kann man z. B. die Fibrillen als Kern eines Kernleiters auffassen. Da wir nun aber wissen, dass nur die Neurofibrillen das ganze Nervensystem durchziehen, während ihre Umgebung wechselt, so käme man zu dem Schluss, dass die Hülle des Kernleiters kein spezifisches Element ist, sondern bald im Nervenfaserplasma, bald im Protoplasma der Ganglienzellen, bald in irgendeiner anderen Leibessubstanz bestehen kann. Die theoretische Forderung beim Kernleiter geht ja auch nur dahin, dass die Hülle von einer wenigstens zum Teil ionisierten Elektrolytlösung gebildet wird.

Trotzdem nach meinen Versuchen die Fibrille als Träger der Leitungsfunktion angesehen werden muss, so kann die von Jenkins und Carls on vertretene Ansicht, dass die leitende Substanz sich wie eine Flüssigkeit verhält oder, allgemeiner gesagt, eine gleichmässige und leicht versehiebliche Molekularstruktur besitzt, doch richtig sein. Ein Beweis dafür liegt allerdings nicht vor, aber da wir über die Konsistenz und den molekularen Aufbau der Fibrillen nichts wissen, so ist es doch möglich. Will man diese Frage auf dem von Jenkins und Carlson beschrittenen Wege zur Entscheidung bringen, so darf man nicht den Nerven innerhalb der physiologischen Längendifferenzen untersuchen, wie diese Autoren es getan haben, sondern muss als Ausgangslänge weiterer Dehnungen die Länge benutzen, bei der die Fibrillen gerade bis zu ihrer natürlichen Länge gedehnt sind. Die wenigen Zahlen, die ich bei Dehnung über die natürliche Länge hinaus erhalten habe, lassen eine Gesetzmässigkeit noch nicht erkennen. Ehe solche Untersuchungen durchgeführt werden können, müsste auch erst noch genauer festgestellt werden, bei welcher Nervenlänge die Fibrillen geradlinig verlaufen. Da wenigstens beim Blutegel schon bei geringer Überdehnung Leitungsunfähigkeit eintritt, so dürften derartige Untersuchungen auf Schwierigkeiten stossen. Dieser frühzeitige Eintritt von Leitungs- 
unfähigkeit bei Dehnung über die natürliche Länge, der auch von Jenkins und Carlson erwähnt wird, liesse sich sogar gegen die Annahme einer gleichmässigen oder leichtverschieblichen Molekularstruktur der leitenden Substanz anführen. Da aber die Möglichkeit besteht, dass die Nerven hierbei nicht überall gleichmässig gedehnt werden, so möchte ich dem Befund nach dieser Richtung hin keinen besonderen Wert beilegen.

Für die flüssige oder halbflüssige Natur der leitenden Substanz wird auch von $\operatorname{Hermann}^{\mathbf{1}}$ ) der von ihm entdeckte Austritt von Substanz am Anodenende eines von einem starken Strom durchflossenen Nerven angeführt. Seitdem sich die Frage dahin verändert hat, oh der Achsenzylinder als Ganzes oder nur vermittelst einer seiner Komponenten leitet, sind aus diesem Versuch für die vorliegende Frage keine Schlüsse mehr zu ziehen.

Nach dem hier vorgebrachten hat die Frage nach der Leitungsgeschwindigkeit des Nerven nur noch bei solchen Nerven einen Sinn, welche eine nahezu konstante Länge haben. Bei Nerven von sehr variabler Länge, wenigstens bei denen des Blutegels, ist die Leitungsgesehwindigkeit des Nerven unter anderem eine Funktion des Dehnungszustandes, d. h. sie wechselt bei jedem Schritt des Tieres $z$ wischen $a$ und etwa $3 a$. Bei solchen Tieren wird man vielmehr die Leitungsgeschwindigkeit der Fibrillen messen müssen und diese bei grösster physiologischer Länge des Nerven bestimmen können, bei der die Fibrillen wenigstens in den bisher untersuchten Tieren geranlinig verlaufen. Als Leitungsgeschwindigkeit der Fibrillen ergibt sich für den Blutegel 32-40 cm in der Sekunde.

\section{Resultate.}

1. In dehnbaren Nerven (Hirudo) ist die Leitungsgeschwindigkeit in einem gegebenen Nervenstück proportional seiner jeweiligen Länge, so lange die Dehnung innerhalb der physiologischen Grenzen bleibt. Da die Neurofibrillen innerhalb der physiologisehen Dehnungsgrenzen die gleiche Länge bewahren, so sind dieselben als die Hauptträger der Erregungslejtung anzusehen.

1) Pflüger's Arch. Bd. 67 S. 240,1897 u. Bd. 70 S. 520. 1898. Ausserdem Jahresbericht 1904 S. 25. 
36 Albrecht Bethe: Ein neuer Beweis für die leitende Funktion etc.

2. Das Nervenfaserplasma (die Perifibrillärsubstanz) hat auf die Geschwindigkeit der Erregungsleitung keinen Einfluss. Hinge die Leitungsgeschwindigkeit nämlich vom Nervenfaserplasma ab, so müsste die Leitungsgeschwindigkeit bei der Dehnung des Nerven konstant bleiben, da der plasmatische Teil der Nervenfasern seine Länge proportional der Dehnung verändert.

3. Die Länge der Reflexlatenz und Muskellatenz ist bei Hirudo abhängig vom Tonuszustand der reagierenden Muskeln.

4. Die Reflexzeit beträgt bei Hirudo höchstens 0,003 Sekunden, ist aber wahrscheinlich geringer.

5. Die Latenz der Reflexhemmung ist bei Hirudo wesentlich grösser als die Latenz der Reflexkontraktion. Mit anderen Worten: Tonusanstieg folgt schneller auf den auslösenden Reiz als Tonusfall.

6. Die Leitungsgeschwindigkeit der Neurofibrillen beträgt beim Blutegel $32-40 \mathrm{~cm}$ in der Sekunde. 